\title{
Hippo Pathway Effectors Control Cardiac Progenitor Cell Fate by Acting as Dynamic Sensors of Substrate Mechanics and Nanostructure
}

\begin{abstract}
Diogo Mosqueira, ${ }^{\dagger, \mp, \wedge}$ Stefania Pagliari, ${ }^{\dagger, \S, \wedge}$ Koichiro Uto, $^{\dagger}$ Mitsuhiro Ebara, $^{\dagger}$ Sara Romanazzo, ${ }^{\dagger}$ Carmen Escobedo-Lucea, ${ }^{\perp}$ Jun Nakanishi, ${ }^{\dagger}$ Akiyoshi Taniguchi, ${ }^{\dagger}$ Ornella Franzese, ${ }^{\|}$Paolo Di Nardo," Marie José Goumans, ${ }^{\#}$ Enrico Traversa, ${ }^{\triangle}$ Perpetua Pinto-do-Ó, ${ }^{\star}$ Takao Aoyagi, $^{\dagger, \nabla, *}$ and Giancarlo Forte ${ }^{\dagger, \nabla, \Lambda, \square, *}$

${ }^{\dagger}$ Biomaterials Unit, International Center for Materials Nanoarchitectonics (MANA), National Institute for Materials Science (NIMS), Tsukuba, Japan, ${ }^{\ddagger}$ Instituto de Engenharia Biomédica-INEB, Universidade do Porto, Porto, Portugal, ${ }^{\S}$ Japan Society for the Promotion of Science (ISPS), Tokyo, Japan, ${ }^{\perp}$ Academy of Finland Research Fellow, Division of Pharmaceutical Biosciences/Center for Drug Research (CDR), University of Helsinki, Helsinki, Finland, "Department of Systems Medicine, University of Rome Tor Vergata, Rome, Italy, ${ }^{\circ}$ Department of Clinical Sciences and Translational Medicine, University of Rome Tor Vergata, Rome, Italy, ${ }^{\# D e p a r t m e n t ~}$ of Molecular Cell Biology, Leiden University Medical Center, Leiden, The Netherlands, ${ }^{\triangle}$ King Abdullah University of Science and Technology (KAUST), Thuwal, Saudi Arabia, and ${ }^{\nabla}$ International Clinical Research Center (ICRC), Integrated Center of Cellular Therapy and Regenerative Medicine, St. Anne's University Hospital, Brno, Czech Republic. ${ }^{\boldsymbol{\Delta}}$ These authors contributed equally. ${ }^{\boldsymbol{}}$ These authors contributed equally. ${ }^{\square}$ Present address: International Clinical Research Center (ICRC), Integrated Center of Cellular Therapy and Regenerative Medicine, St. Anne's University Hospital, Brno, Czech Republic.
\end{abstract}

\begin{abstract}
Stem cell responsiveness to extracellular matrix (ECM) composition and mechanical cues has been the subject of a number of investigations so far, yet the molecular mechanisms underlying stem cell mechano-biology still need full clarification. Here we demonstrate that the paralog proteins YAP and TAZ exert a crucial role in adult cardiac progenitor cell mechano-sensing and fate decision. Cardiac progenitors respond to dynamic modifications in substrate rigidity and nanopattern by promptly changing YAP/TAZ intracellular localization. We identify a novel activity of YAP and TAZ in the regulation of tubulogenesis in 3D environments and highlight a role for YAP/TAZ in

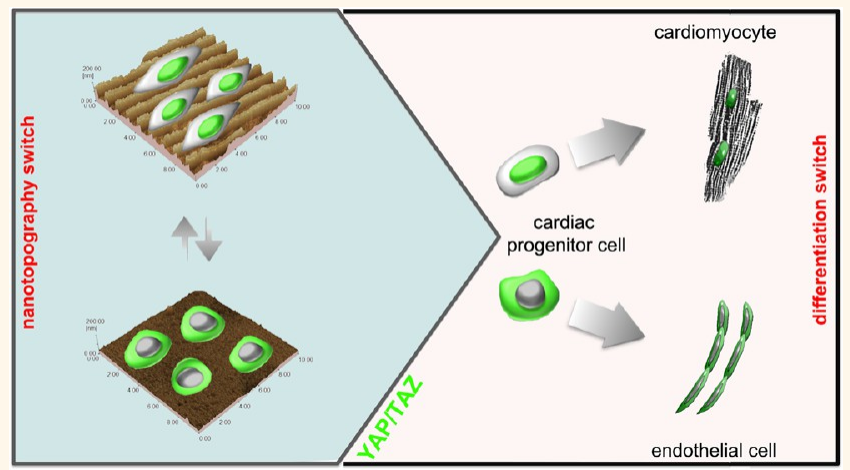
cardiac progenitor proliferation and differentiation. Furthermore, we show that YAP/TAZ expression is triggered in the heart cells located at the infarct border zone. Our results suggest a fundamental role for the YAP/TAZ axis in the response of resident progenitor cells to the modifications in microenvironment nanostructure and mechanics, thereby contributing to the maintenance of myocardial homeostasis in the adult heart. These proteins are indicated as potential targets to control cardiac progenitor cell fate by materials design.
\end{abstract}

KEYWORDS: adult cardiac progenitor cell $\cdot$ substrate nanotopography $\cdot$ mechano-transduction $\cdot$ cardiac differentiation $\cdot$ YAP/TAZ

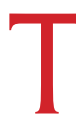
he Hippo pathway has been recently identified as a crucial axis in the regulation of organ size and shape during organogenesis and cancer. The paralog Yes-associated protein (YAP) and WW domain-containing transcription regulator protein 1 (WWTR1 or TAZ) are the downstream effectors of the Hippo pathway. These proteins have also been identified as mammalian proto-oncogenes. ${ }^{1}$ Moreover, they perform as transcriptional coactivators in the nucleus, mainly in combination with transcription factors belonging to
TEAD family. ${ }^{2}$ In vitro, YAP/TAZ activity has been associated with mesenchymal stem cell (MSC) fate decision through the interaction with key determinants of osteogenic (Runx2) or adipogenic (PPAR $\gamma$ ) differentiation.,

YAP/TAZ axis has also emerged as a central regulator of human embryonic stem cell self-renewal through the control of SMAD complex shuttling to the nucleus, with TAZ knock-down resulting in the loss of cell pluripotency. ${ }^{5}$ The same cofactors control intestinal ${ }^{6}$ and neural progenitor cell number and differentiation ${ }^{7}$ by targeting

\section{* Address correspondence to giancarlo.forte@fnusa.cz aoyagi.takao@nims.go.jp.}

Received for review June 16, 2013 and accepted February 2, 2014.

Published online

10.1021/nn4058984

(c) XXXX American Chemical Society 
the Notch signaling. YAP activity has also been proven fundamental in controlling epidermal stem cell homeostasis. ${ }^{8}$ Finally, TAZ is a critical determinant in skeletal muscle differentiation since its nuclear localization is required for myofiber formation, ${ }^{9,10}$ while its activation through the Hippo pathway inhibition increases induced pluripotent stem cell (iPS) generation from somatic cells. ${ }^{11}$

The composition and the mechanical properties of the substrate are critical parameters in stem cell maintenance and differentiation. MSCs can sense the stiffness of the underlying substrate, and thus can have their fate driven by changes in matrix mechanical properties. $^{12}$ The differentiation capacity of muscle progenitors is hindered by high substrate stiffness, ${ }^{13}$ while the assembly of functional contractile structures in embryonic cardiomyocytes requires appropriate matrix elasticity. ${ }^{14}$ The importance of substrate compliance in embryonic stem cell (ES) growth and pluripotency has been recently demonstrated, with YAP/TAZ identified as key factors sensing substrate mechanics and driving cell fate. ${ }^{15}$

The YAP/TAZ subcellular localization, strictly regulated by cell-cell and cell-extracellular matrix (ECM) interaction, has been correlated to cell mechanosensing. ${ }^{16}$ In particular, YAP/TAZ show a preferential cytoplasmic localization when cells can sense cell-tocell contact, while moving into the nucleus when single cells are seeded on stiff substrates. This evidence implies that YAP/TAZ intracellular localization and activity are tightly regulated by cytoskeleton arrangement and cell tension. ${ }^{17}$ In fact, YAP/TAZ have recently been described as general relays in cell mechano-sensing, shuttling to the nucleus when the substrate becomes stiffer and cells can spread assembling their cytoskeleton. ${ }^{18}$ As a matter of fact, ECM elastic properties vary significantly between tissues, and a number of pathological conditions have been associated with changes in this parameter. $^{19}$

The increase in heart size occurring during embryogenesis, a process mainly due to cardiac progenitor cell and fetal cardiomyocyte proliferation, is paralleled by an increase in myocardium stiffness reaching physiological values around $10 \mathrm{kPa}$ in the adult heart. This value can locally rise up to $35-70 \mathrm{kPa}$ in the infarcted myocardium. ${ }^{20}$ On the other hand, heart growth after birth is largely due to size increase of postnatal cardiomyocytes, that is, physiological hypertrophy. Interestingly, the Hippo pathway has been identified as a critical determinant of the organ size by controlling fetal cardiomyocyte proliferation, ${ }^{21}$ through the IGF axis $^{22}$ or by inhibiting the $\mathrm{Wnt} / \beta$-catenin signaling pathway. ${ }^{23}$ Cardiomyocyte-specific YAP1 deletion in the fetal mouse heart causes lethal myocardial hypoplasia and a reduction in cardiomyocyte proliferation, while this factor appears to be dispensable for the postnatal physiological hypertrophy. ${ }^{21}$
Cardiac tissue homeostasis throughout life is thought to depend on the proliferation and differentiation of resident stem/progenitor cells, ${ }^{22}$ although their actual capacity to enhance organ function is still debated. These cells have been shown to slowly replenish cardiomyocytes and vascular cells ${ }^{24}$ when needed and their maintenance to be tightly regulated by biological and mechanical cues arising from the surrounding cells and ECM. $^{25}$

In the present study, we identified the crucial role of YAP/TAZ activity in Sca- $1^{+}$human cardiac progenitor cell migration, proliferation, and differentiation. In this particular cardiac cell subset, which has been shown to acquire cardiac and endothelial phenotype in vivo, ${ }^{26}$ we show that YAP/TAZ intracellular localization is a direct function of substrate elasticity in a physiologically relevant range $(0.5-40 \mathrm{kPa})$ and depends on cell spreading as a result of cytoskeleton organization. Moreover, by mimicking the modifications occurring after myocardial infarction in cardiac ECM through smart thermo-responsive materials, we provide evidence that YAP/TAZ dynamically respond to changes in substrate nanostructure and elasticity. Furthermore, they also control Sca- $1^{+}$cardiac progenitor ability to produce tubular structures on a soft environment and migrate on stiff substrates. Finally, we show that YAP/TAZ exert a key role in controlling human cardiac progenitor cell differentiation toward endothelial or cardiomyocyte lineage as induced by biological supplements or substrate composition in vitro.

Together with our observation that YAP/TAZ expression is induced in cardiac cells at the infarct border zone, these results highlight a direct role for such proteins in adult cardiac tissue response to ECM modifications.

\section{RESULTS}

YAP/TAZ Are Mechano-Sensors in Sca- ${ }^{+}$Cardiac Progenitor Cells and Their Expression Is Triggered at the Infarction Border Zone. The expression of YAP/TAZ transcriptional coactivators has been shown to regulate cardiomyocyte proliferation and cardiac morphogenesis during fetal life. ${ }^{21}$ In good agreement with what was recently demonstrated in the adult healthy myocardium, ${ }^{27}$ YAP/ TAZ expression is hardly detectable in adult cardiomyocytes (Figure 1a). Interestingly, in the infarcted myocardium, YAP/TAZ expression could be detected in the border zone at days 3, 5, 14, and 36 after myocardial infarction (MI), while cardiomyocytes in remote areas were faintly stained by the YAP/TAZ-specific antibody (Supplementary Figure 1a,b).

The occurrence of myocardial infarction is associated with an increase in ECM stiffness ${ }^{20}$ and severe modifications in ECM nanostructure. ${ }^{19}$ Along with the occurrence of a severe inflammatory response, the loss in ECM compliance associated with myocardium 

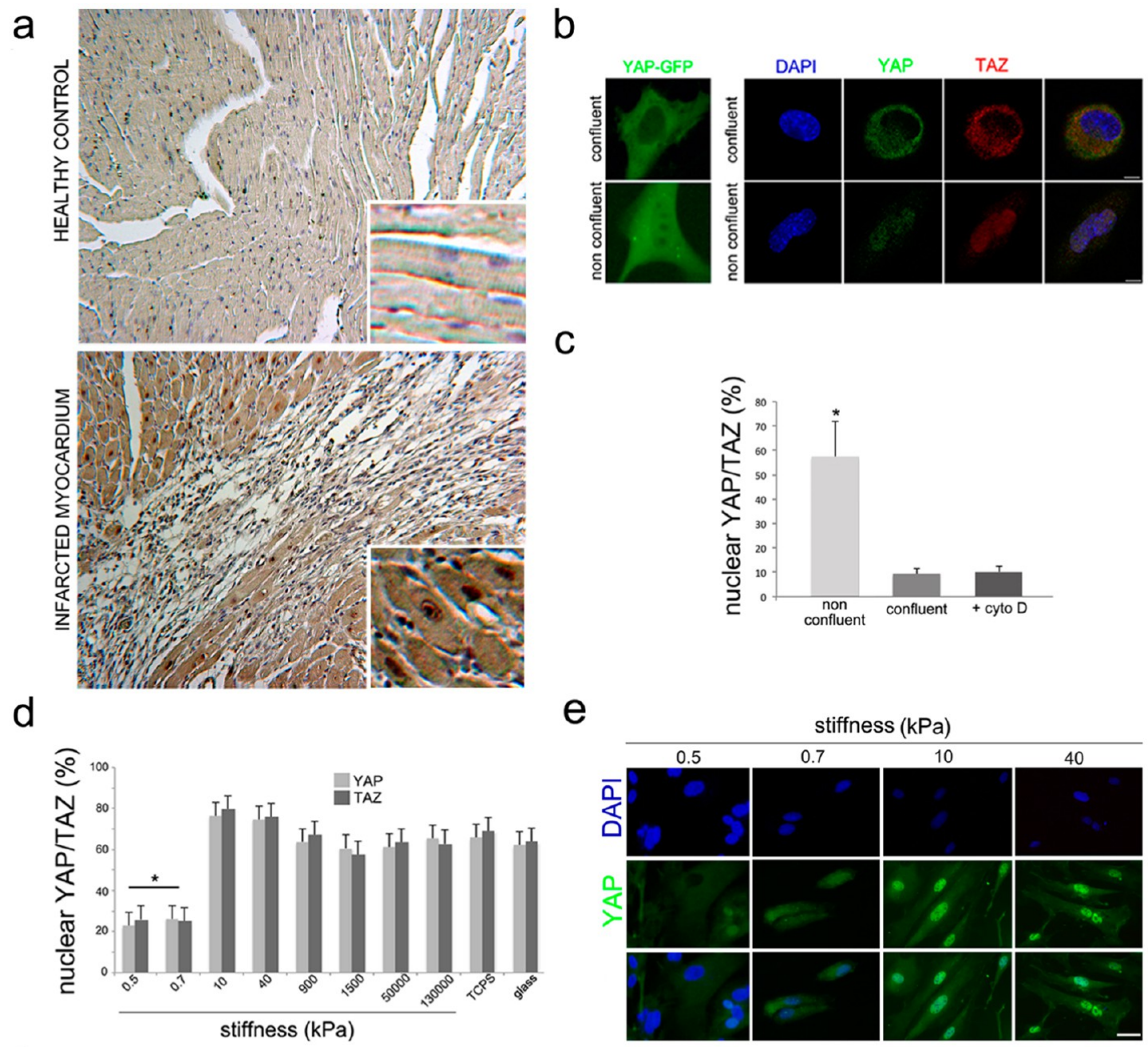

f

Thermo-responsive PCL polymer

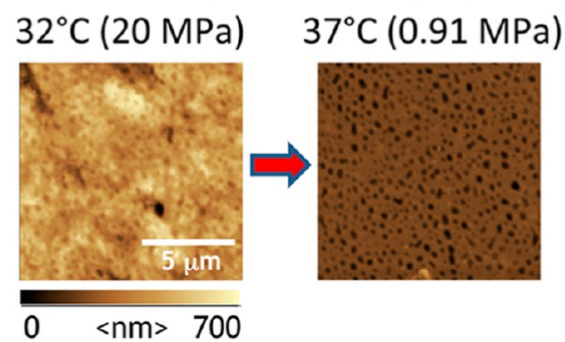

g

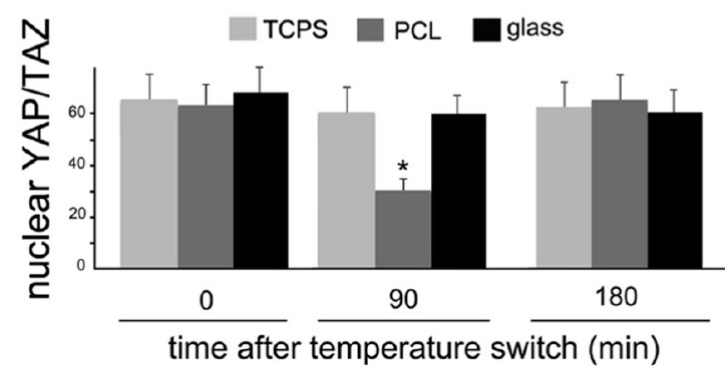

Figure 1. YAP/TAZ intracellular localization is dynamically regulated in cardiac progenitor cells as a function of substrate stiffness. YAP/TAZ expression is nearly undetectable in healthy adult murine heart, while its expression is triggered in cardiac cells at the infarction border zone (a). The images are representative of 12 random fields taken at day 5 after myocardial infarction. Scale bar: $200 \mu \mathrm{m}$. YAP/TAZ can be detected inside the nucleus in nonconfluent cardiac progenitor cells, while shuttling to the cytoplasm when such cells are confluent, as demonstrated by YAP-GFP transduction or immunostaining with anti-YAP antibody (b). YAP/TAZ display nuclear localization in sparse but not in confluent cells. This relocalization requires cytoskeleton integrity, as demonstrated through cytochalasin D-induced disruption of F-actin assembly triggering YAP relocalization to the cytoplasm (c). See also Supplementary Figure $1 \mathrm{~b}$ (Supporting Information). YAP/TAZ shuttling to the nucleus is sensitive to substrate stiffness, their expression being stained in the nucleus when $E \geq 10 \mathrm{kPa}(24 \mathrm{~h})(\mathrm{d}, \mathrm{e})$. When thermo-responsive $\mathrm{PCL}$ polymers are induced to a conformational shift by temperature switch (f), YAP/TAZ intracellular localization is dynamically modified (g). YAP/TAZ relocalization could not be ascribed to temperature, as demonstrated by culturing the cells onto tissue culture polystyrene (TCPS) or glass dishes. For more details on substrate mechanical properties, see Supplementary Figure 1e. ${ }^{*} P<0.05$, in (d) $P: \mathrm{kPa}$ group vs MPa group. All experiments were performed in triplicate, yielding similar results $(n=3)$.

remodeling has been described as one of the main limitations to resident stem/progenitor cell activation and differentiation. ${ }^{28,29}$ The effect of increased substrate stiffness on cardiomyocyte activity has been already reported by our group and others; ${ }^{14,30}$ thus, we hypothesized that substrate stiffening could affect 
cardiac progenitor cell function and contribute to the onset of heart failure.

To follow YAP cellular localization, human cardiac progenitors were engineered to express green fluorescent protein (GFP) under YAP promoter (YAP-GFP hCMPCs). The cells showed prevalent GFP expression in the cytoplasm when confluent, while a predominant nuclear presence was found in nonconfluent cells. The data were confirmed by confocal immunostaining (Figure $1 \mathrm{~b}, \mathrm{c}$ and Supplementary Figure 1c). Similar results were obtained on murine $\mathrm{Sca}-1^{+}$cardiac progenitor cells and neonatal cardiomyocytes (Supplementary Figure 2).

YAP/TAZ localization in hCMPCs was clearly dependent on cytoskeleton integrity since its disruption by cytochalasin D (cyto D) treatment triggered their relocalization into the cytoplasm (Figure 1c and Supplementary Figure 1c). A similar effect on YAP expression could be induced by $4 \mathrm{~h}$ cell treatment with blebbistatin (blebb)—inhibiting myosin II contractility-and Rho A/ROCK inhibitor Y27362. Both inhibitors block the transmission of tension and remodel cytoskeleton arrangement (Supplementary Figure 1f). The effect was transient and could be reverted after $6 \mathrm{~h}$ washout (Supplementary Figure 1g).

To test the possibility that hCMPCs utilize YAP/TAZ as a relay for substrate stiffness, collagen- and fibronectin-coated polyacrylamide (PA) gels with different PA concentrations were prepared as previously described. $^{31}$ The films displayed physiological elastic moduli $(E)$ in the $\mathrm{kPa}$ range and were compared to $P C L$ cross-linked polymers with increasing $E$ values in the MPa range, similar to those previously reported. ${ }^{32}$ Under these conditions, YAP/TAZ displayed a selective sensitivity to substrate stiffness in the $\mathrm{kPa}$ range. The elastic modulus values are similar to those encountered in living tissues in physiological and pathological conditions. Twenty-four hours after seeding, YAP/TAZ were predominantly located in the cytoplasm on PA films showing very low stiffness (resembling fat tissue: $0.5-0.7 \mathrm{kPa}$ ), while shuttling to the nucleus when $E \geq 10 \mathrm{kPa}$. Interestingly, no significant changes in YAP/TAZ subcellular localization could be found on supra-physiological stiff substrates. In these conditions, the factors were predominantly stained in the nucleus (Figure 1d,e). These data indicated that cardiac progenitor mechano-sensitivity applies only in the physiological range of stiffness and that the process of mechano-sensing starts as early $(3-5 \mathrm{~h})$, as the cells contact the substrate (Supplementary Figure 2c). Finally, PCL thermo-responsive films having a melting temperature $\left(T_{\mathrm{m}}\right)$ between 32 and $37^{\circ} \mathrm{C}$ were induced to a stiffness switch by changing the temperature. YAP/ TAZ expression was followed in hCMPCs during the thermal transition. As expected, YAP/TAZ expression was mainly detected in the nucleus of hCMPCs cultured on stiff $\mathrm{PCL}$ films at $32{ }^{\circ} \mathrm{C}\left(E_{32^{\circ} \mathrm{C}}=20 \mathrm{MPa}\right)$.
Following the stiffness switch $\left(E_{37^{\circ} \mathrm{C}}=0.9 \mathrm{MPa}\right)$, a significant decrease in nuclear YAP/TAZ expression occurred. Remarkably, the percentage of YAP nuclear positive cells returned to the values found before the temperature switch within $180 \mathrm{~min}$ (Figure 1f,g). The mechanical properties of the thermo-responsive polymer used are thoroughly described in Supplementary Figure 1e.

YAP/TAZ Intracellular Localization Is Sensitive to Cardiac Progenitor Cell Spreading and Independent of Cell Shape. Substrate stiffness has been correlated with the ability of cells to adopt a given morphology while spreading on the surface, independently of the cell-ECM contact area. ${ }^{18}$ To study YAP/TAZ dependency on cell spreading and confinement, we grew hCMPCs onto micropatterned fibronectin surfaces with different areas. In this experimental setting, cells spread onto larger islands $\left(2025,10000 \mu \mathrm{m}^{2}\right)$ where actin bundles could be detected, whereas onto smaller islands (300, $1024 \mu \mathrm{m}^{2}$ ), each cell appeared confined (Figure 2a). This single cell system allowed the identification of a gradient in YAP/TAZ nuclear shuttling already $3-5 \mathrm{~h}$ after seeding (Supplementary Figure 3a). After 24 h, YAP/TAZ localized primarily in the nucleus of spread hCMPCs, while in cells grown on the smaller islands, their expression was predominantly cytoplasmic (Figure 2a). A similar behavior was detected in murine $\mathrm{Sca}-1^{+}$cardiac progenitor cells (Supplementary Figure $3 b, c$ ), thus pointing at YAP/TAZ shuttling as a general mechanism for substrate sensing in cardiac resident progenitors. Given the sensitivity of YAP/TAZ localization to cytoskeleton integrity, we hypothesized that differences in cell polarity could affect the activity of these factors. Thus, we designed micropatterned fibronectin-coated arrays in which cell shape could be controlled (squares, circles, triangles) by the availability of the adhesion sites. By this means, the cells would have the same surface area $\left(2000 \mu \mathrm{m}^{2}\right)$ but acquire different shapes (Figure $2 b, c$ ), and we demonstrated that YAP expression was independent from cell shape, while being controlled by the surface area.

Although cell shape and spreading has been lately shown to control osteogenic versus adipogenic differentiation in human mesenchymal stem cells (hMSCs), ${ }^{33}$ the expression of early (GATA-4, Figure $2 d$ ) or late (alpha sarcomeric actinin, Supplementary Figure 3d) differentiation markers was not modified by hCMPCs spreading onto micropatterned fibronectin islands.

YAP/TAZ Are Required for Cardiac Progenitor Cell Adhesion and Migration on Stiff Substrates. The role of YAP/TAZ in different cell types has been associated with their acknowledged activity as transcriptional coactivators, thus modulating the expression of genes involved in a number of specific responses. ${ }^{2}$ YAP/TAZ nuclear activity seems to be mostly required for cardiac progenitor cell adhesion onto stiff substrates $(E \geq 10 \mathrm{kPa})$, as demonstrated by the AlamarBlue assay, giving a 

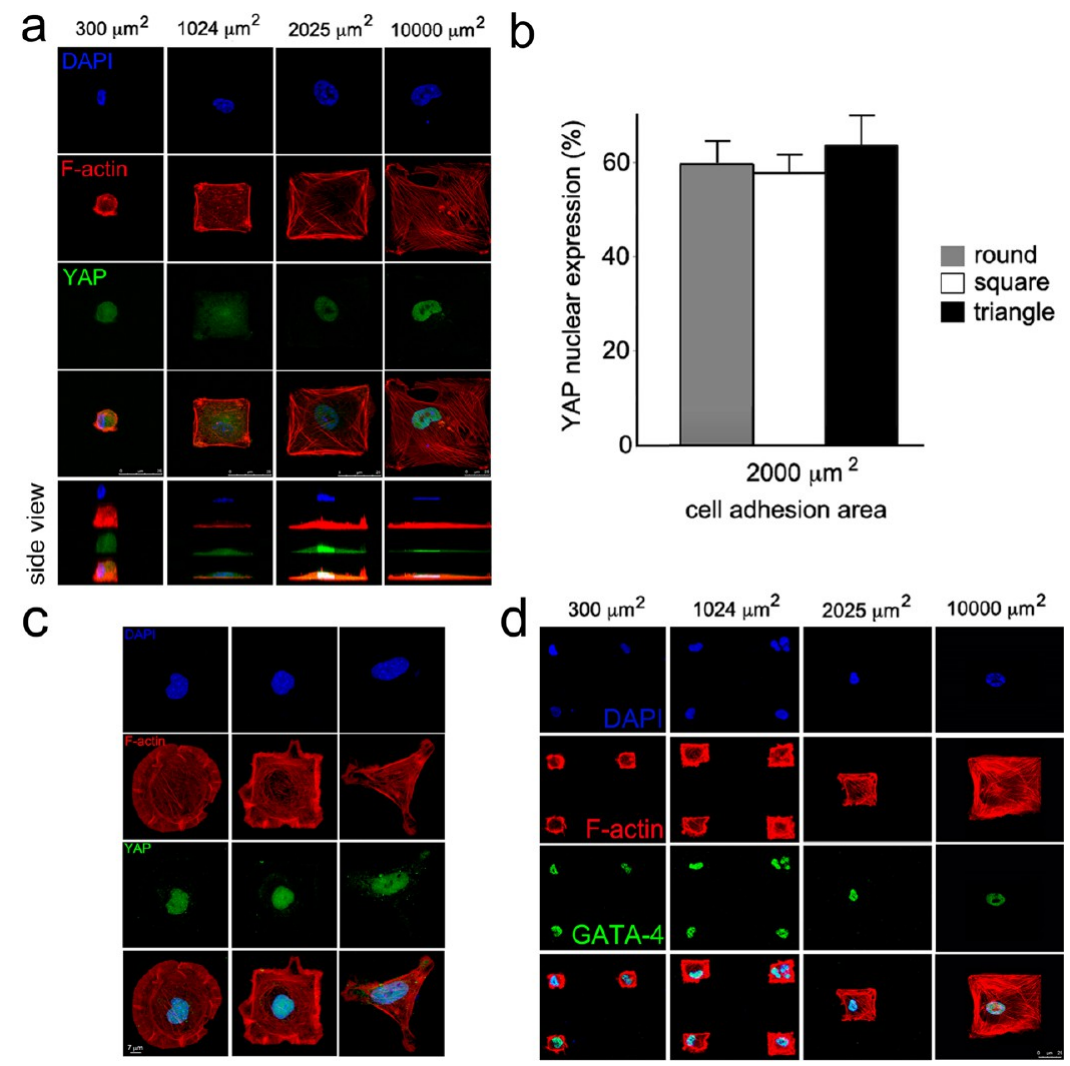

Figure 2. YAP/TAZ sense cell spreading and cytoskeleton organization in cardiac progenitor cells. YAP/TAZ intracellular localization in response to cell spreading on fibronectin micropatterned square islands of increasing area $(300,1024,2025$, and $10000 \mu \mathrm{m}^{2}$ ) suggests a gradient in the intracellular localization of such factors as dependent on cell spreading on adhesive surfaces (for a time-course kinetic and quantification of YAP/TAZ expression, see Supplementary Figure 3). YAP/TAZ expression is exclusively nuclear in cardiac progenitors grown onto large islands, while it is confined to the cytoplasm on small islands after $24 \mathrm{~h}$ (a). Micropatterned fibronectin-coated islands were designed to control cell area $\left(2000 \mu \mathrm{m}^{2}\right)$ and shape (squares, circles, and triangles). No differences in YAP expression were detected in cardiac progenitors having same cell area and different shape, thus showing that cell area controls YAP sublocalization independently of cell shape $(b, c)$. No change in GATA-4 early cardiac commitment marker expression could be detected as an effect of cell spreading in human cardiac progenitors (d). The data represent $n=3$ independent experiments being performed in duplicate.

meaningful estimation of differences in cell adhesion rate (Figure 3a). This dissimilar cell behavior can be easily ascribed to the massive shift in the expression of genes involved in cell-matrix interaction (collagens, laminins, integrins) observed in cells in which YAP/ TAZ were silenced (siYAP, Figure $3 b$ ). Given that YAP/ TAZ nuclear activity seems to be important for the adhesion of hCMPCs onto stiff matrices, photoactivated glass surfaces $(E=\infty)^{34}$ were prepared in which cells are confined in $900 \mu \mathrm{m}^{2}$ adhesive areas, without the possibility to spread (Figure $3 c$ and Supplementary Figure 4c). As expected, in this condition, YAP/TAZ staining was mainly detected in cell cytoplasm, despite substrate stiffness being virtually infinite. Selective UV light $(\lambda: 365 \mathrm{~nm})$ irradiation of the surface allowed the cells to spread and migrate out of the confined areas (Supplementary movie 1). By following cell migration in time-lapse automated microscopy, a significant shuttling of YAP/TAZ from the cytoplasm to the nucleus was detected (Figure 3d). Notably, YAP/TAZ-silenced cardiac progenitor cells displayed reduced migration capacity, thus showing that
YAP/TAZ axis is required for hCMPC migration on stiff substrates.

YAP/TAZ in Cardiac Progenitor Cells Are Sensitive to Dynamic Changes in Substrate Nanotopography. Given the selective relocalization of YAP/TAZ in response to modifications in substrate mechanics, we reasoned that these proteins could be sensitive to dynamic changes in matrix nanostructure, thus acting as an on-off relay. Thermo-responsive cross-linked PCL polymers displaying shape-memory properties in response to slight temperature modifications ${ }^{32}$ were fabricated in an attempt to mimic the modifications in ECM nanostructure following myocardial infarction. ${ }^{19} \mathrm{~A}$ controlled nanopattern was printed on the films at $32{ }^{\circ} \mathrm{C}$ (line width $=300 \mathrm{~nm}$; spacing $=500 \mathrm{~nm}$; line height $=$ $120 \mathrm{~nm}$; Figure 4a) that could be dynamically removed by switching the temperature to $37{ }^{\circ} \mathrm{C}$ (Figure $4 \mathrm{~b}$ and Supplementary movie 2). A significant shift in YAP/TAZ nuclear expression could be detected after $90 \mathrm{~min}$, when the surface nanopattern was modified by the temperature switch (Figure 4c). The percentage of cells expressing nuclear YAP/TAZ 
a

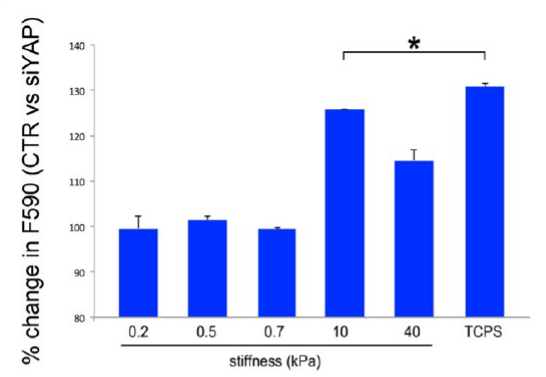

C

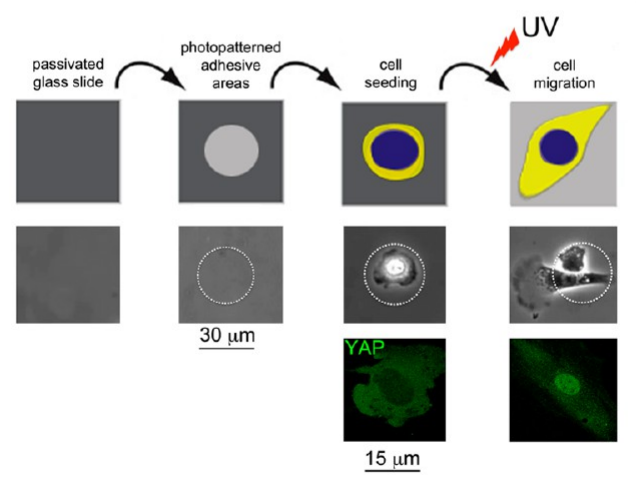

b

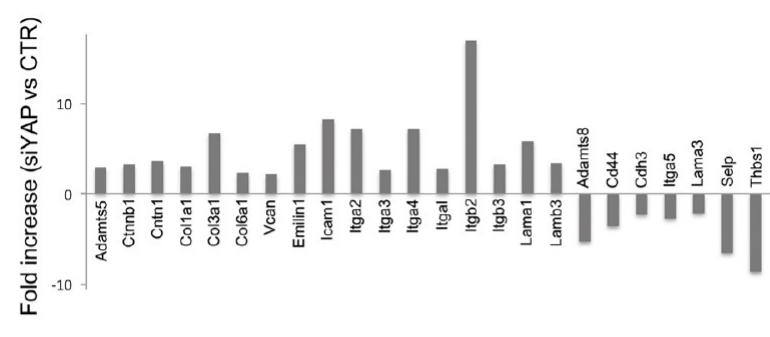

d
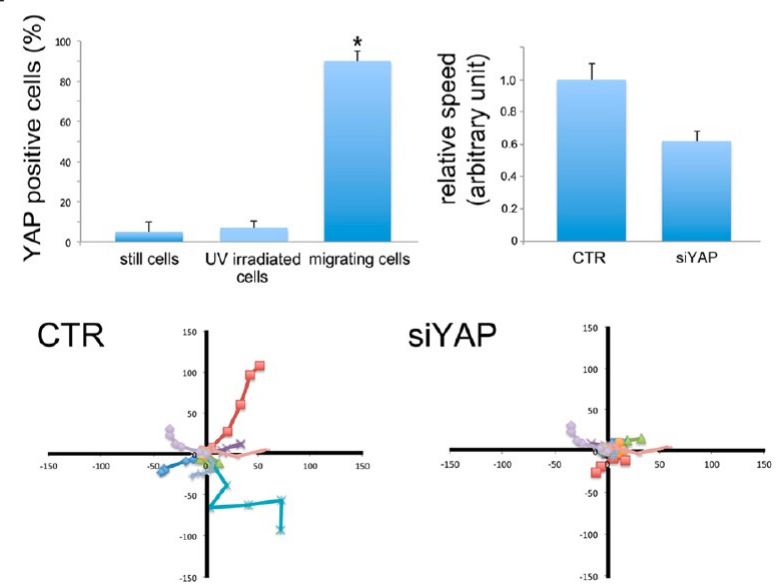

Figure 3. YAP/TAZ nuclear expression is required for cardiac progenitor cell adhesion and migration onto stiff substrates. AlamarBlue assay has been used to quantify cell adhesion and reveals that YAP/TAZ expression is needed for the adhesion of cardiac progenitor cells on stiff substrates $(E \geq 10 \mathrm{kPa})$, while being nonessential on soft surfaces $(E \leq 0.7 \mathrm{kPa})(\mathrm{a})$. Cells in which the expression of YAP was abolished (siYAP) show a marked remodeling in the expression of genes involved in cellmatrix interaction (b). Photoactivated patterned glass surfaces were prepared to confine single hCMPCs on small rounded adhesive areas $\left(900 \mu \mathrm{m}^{2}\right)$ and to study YAP activity in migrating cells. Confined cells show a characteristic cytoplasmic YAP/TAZ expression (c). When cardiac progenitor cell migration was triggered by UV irradiation (UV), YAP/TAZ translocated to the nucleus in migrating cells (YAP in green). No specific effect of UV light per se independently of cell migration could be detected. As expected, YAP silencing significantly impaired $h C M P C$ ability to migrate (d). ${ }^{*} P<0.05, n=3$.

was restored, approaching the original value after $180 \mathrm{~min}$ (Figure 4d). This dynamic modification is consistent with a cellular response to the modification of substrate nanopattern.

YAP/TAZ Contribute to the Control of Cardiac Progenitor Cell Differentiation by Sensing Substrate Elasticity. YAP/TAZ ability to control the differentiation potential of embryonic $^{5}$ and adult ${ }^{6-10}$ stem cells has been compellingly demonstrated, while the Hippo pathway has been described as a barrier to cellular reprogramming in induced pluripotent stem cells. ${ }^{11}$

In the cardiac system, YAP/TAZ key role in cardiomyocyte proliferation and maturation during prenatal life, ${ }^{23}$ but not in postnatal physiological hypertrophy, ${ }^{21}$ is acknowledged.

Sca-1 expression in cardiac stem/progenitor cells has been associated with the multipotential capacity of these cells, which is lost in long passaged cardiac progenitor cells in culture. ${ }^{35}$

In human cardiac progenitor cells, YAP silencing correlated to a significant increase in cell proliferation, as demonstrated by ki67 expression (Figure 5a and Supplementary Figure 5a), and to a significant enhancement in Sca-1 positivity in long cultured passage (p25) hCMPCs (Figure 5b). The concomitant down-regulation in the expression of genes typical of differentiated cardiomyocytes (actn2, adrb1, ckm, kcnq1, mb, myh7, myl2, myl3, myl7, ryr2, tnni3, tnnt2, nat1) and the up-regulation of endothelial-specific genes (edn1, eng, flt1, id1, kdr, pecam1, pgf, tek) were demonstrated in YAP-silenced cells as compared to the controls (Figure $5 \mathrm{c}$ ). To investigate the role of YAP/TAZ in cardiac progenitor cell differentiation, fibronectincoated polyacrylamide gels having stiffness values similar to those found in patho-physiological conditions in vivo $(0.5,10$, and $40 \mathrm{kPa})$ were seeded with cells expressing green fluorescent protein under cardiacspecific promoter cTNT (cTnT-GFP hCMPCs) or YAPsilenced cells. At standard heart stiffness (i.e., $10 \mathrm{kPa}$ ), hCMPCs more efficiently unfolded the cardiac differentiation program as demonstrated by GATA-4 and Nkx-2.5 increase (Figure 5d) and GFP (cTnT) expression (Figure 5e and Supplementary Figure 5b). Few cells showing cardiac Troponin T (CTNT) staining in structures resembling sarcomeres could be found on such substrate (Figure $5 f$ ). The assembly of the sarcomeres 
a
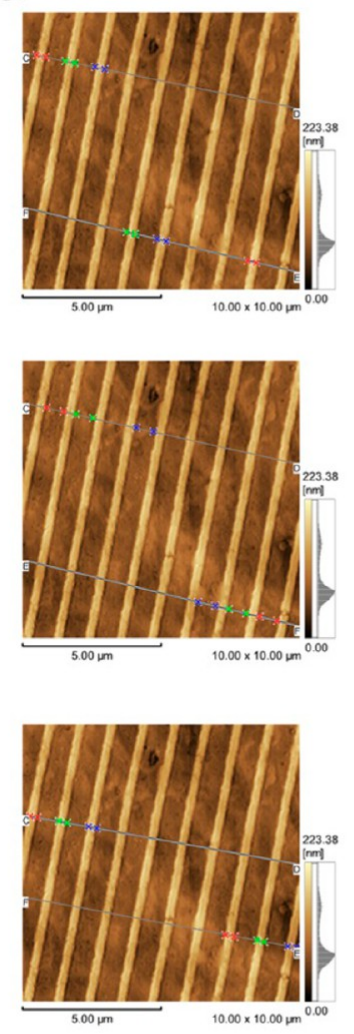
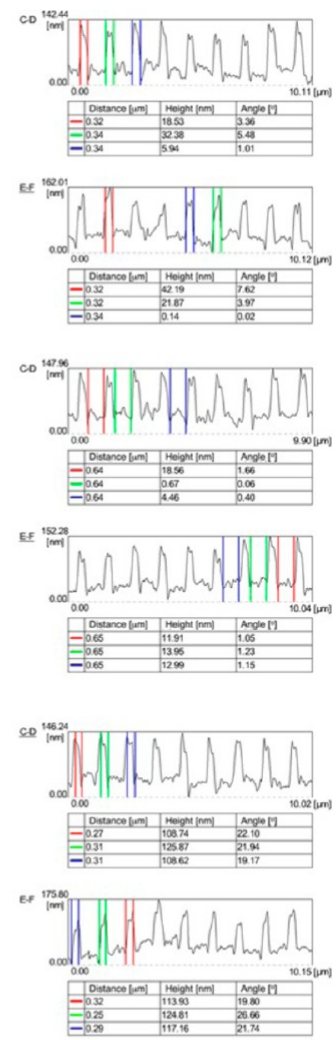

b

temporary nanopattern permanent nanopattern $\left(32^{\circ} \mathrm{C}\right)$

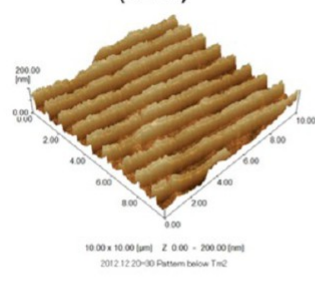

C
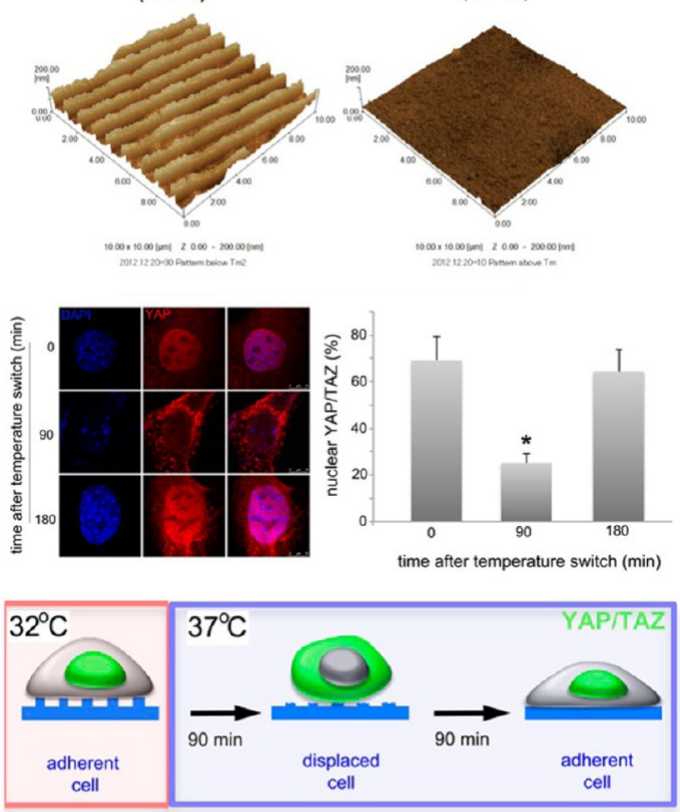

d

temporary nanopattern $\left(32^{\circ} \mathrm{C}\right)$

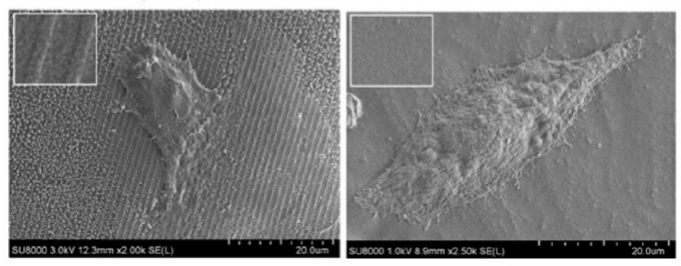

Figure 4. YAP/TAZ nuclear localization is sensitive to dynamic changes in substrate nanopattern in cardiac progenitor cells. PCL-based thermo-responsive substrates having shape-memory effect were produced to display a temporary nanopattern at $32{ }^{\circ} \mathrm{C}$. (a) Topographic surface analysis of nanopatterned PCL films as obtained by atomic force microscopy (AFM). The width (top), space between (middle), and the height (bottom) of the nanolines are shown and compared to the same parameters calculated in the glass mold used. The surfaces could be induced to a thermal transition after switching the temperature to $37{ }^{\circ} \mathrm{C}$ to acquire a permanent nanopattern and mimic the modifications in cardiac ECM nanostructure following myocardial infarction (b). When substrate nanopattern is modified, cells are displaced from the surface and encounter a dramatic but transient switch in YAP/TAZ nuclear expression, which is restored after $180 \mathrm{~min}$ (c). SEM analysis shows hCMPC cells adhering to the surface before (left) and after (right) the thermal transition (d). ${ }^{*} P<0.01, n=3$.

was never complete on PA gels, as expected from previous experiments. ${ }^{25}$ Conversely, YAP silencing significantly hindered the cardiac differentiation in hCMPCs on $10 \mathrm{kPa}$ gels (Figure 5e,f). Alternatively, the expression of YAP/TAZ in CTnT-GFP-expressing murine cardiac progenitors was abolished by shRNA technology and cells induced to differentiate by cardiogenic growth factor stimulation, as previously described. ${ }^{35}$ A clear impairment in CPCs' differentiation ability in cells stably silenced for YAP/TAZ (shYAP) demonstrated that these factors are involved in cardiac progenitor cell differentiation as induced by cardiac induction medium (Figure $5 \mathrm{~g}$ ).

Since a significant up-regulation in genes involved in endothelial commitment was detected in YAP-silenced cells, a meaningful tube formation assay was set up to study YAP and TAZ activity during this process.
Therefore YAP- (siYAP) and TAZ-silenced (siTAZ) cells were prepared and cultured on Matrigel (Figure 6).

As expected for such a soft substrate $(E<1.0 \mathrm{kPa})$, YAP/TAZ expression was exclusively detected in the cytoplasm of hCMPCs, while the cells demonstrated the ability to branch and migrate within the gel (Figure 6a and Supplementary Figure 6a). In YAP/TAZ-silenced cells, the up-regulation of genes encoding for the metalloproteinases (MMPs) 2, 3, 9, 10, 13, and 15 and the concomitant down-regulation of those codifying for tissue inhibitors of metalloproteinases (TIMPs) 1 and 3 could be detected (Figure 6b). As a consequence, YAP/TAZ-silenced cells (siYAP, siTAZ) displayed a more pronounced ability to migrate and produce vessel-like structures. This effect was abrogated by a pretreatment with the broad range MMP inhibitor GM6001 (Figure 6c, d). Interestingly, preincubation with the nuclear 
a
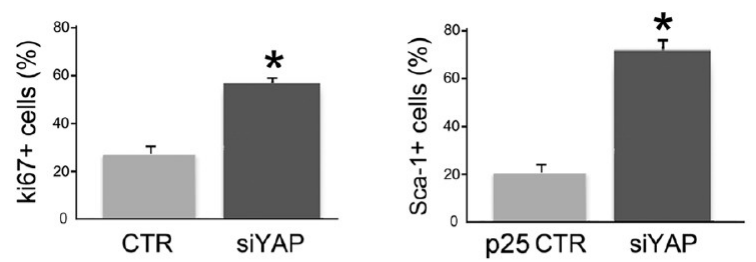

C
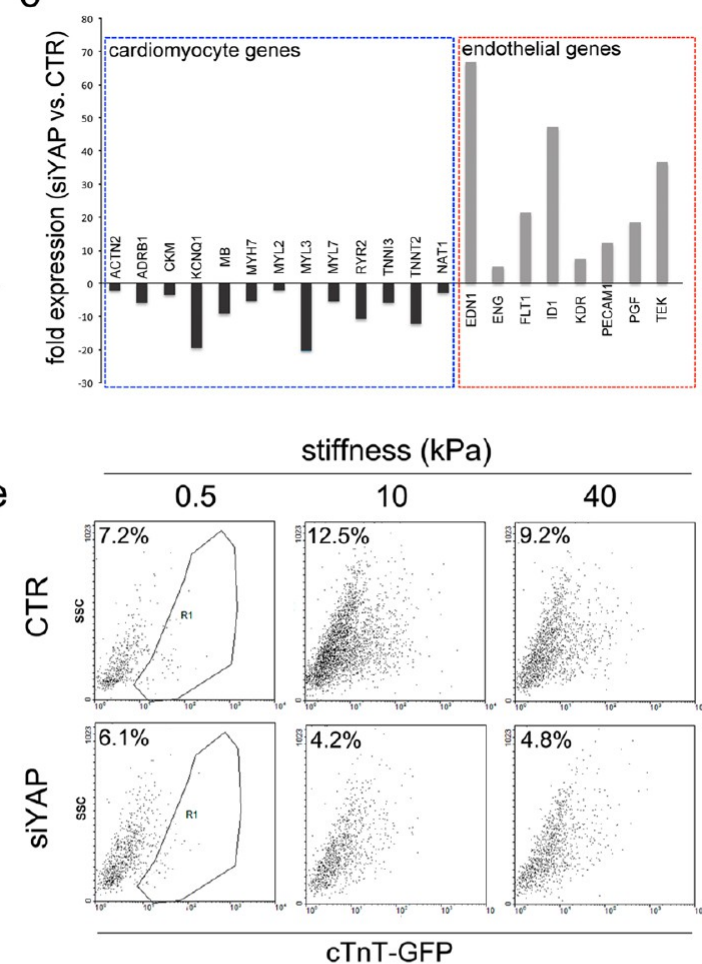

f

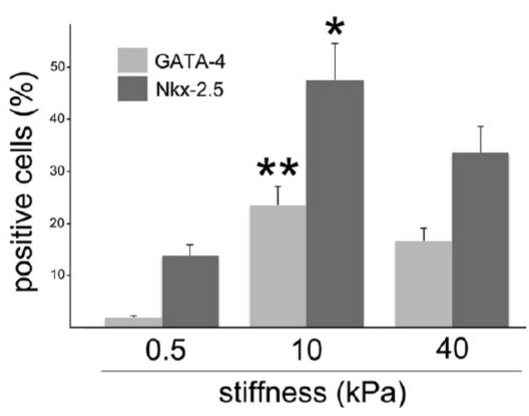

g

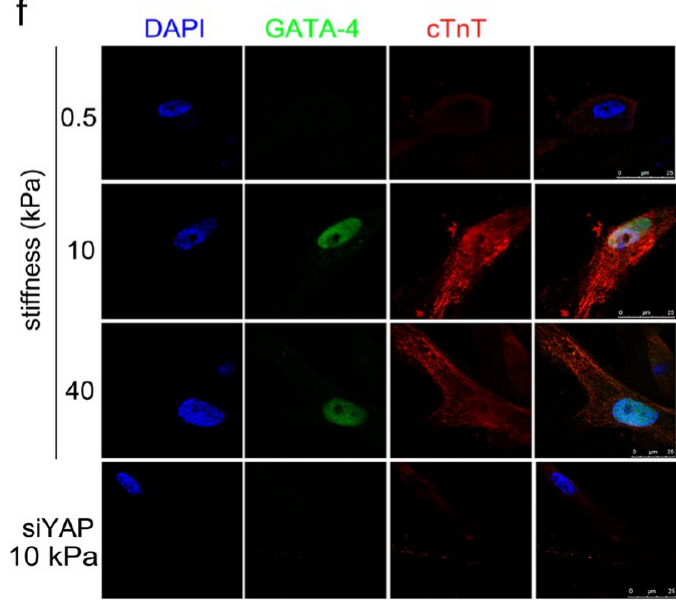
detail
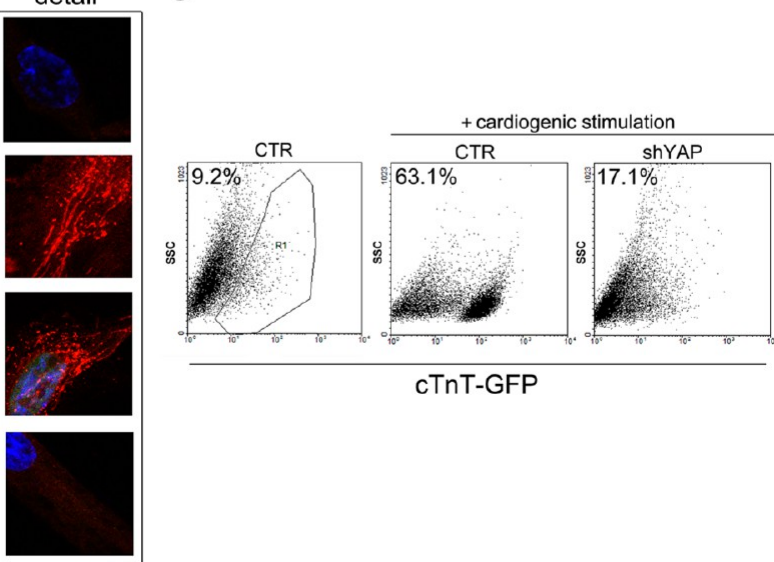

Figure 5. YAP/TAZ contribute to the control of cardiac stem cell proliferation and differentiation. The silencing of YAP/TAZ (siYAP) triggers a significant enhancement in cell proliferation in human cardiac progenitor cells, as assessed by ki67 expression (a), and restores the expression of Sca-1 antigen in long-term passaged cells (p25) (b). YAP-silenced cells display a reduction in the expression of cardiomyocyte-specific genes (actn2, adrb1, ckm, kcnq1, mb, myh7, myl2, myl3, myl7, ryr2, tnni3, tnnt2, nat1) and the concomitant up-regulation in the expression of endothelial genes (edn1, eng, flt1, id1, kdr, pecam1, pgf, tek) (c). ${ }^{*} P<0.05$, between CTR and siYAP ( $n=3)$. Cardiac commitment (as determined by GATA-4 and Nkx-2.5 up-regulation) can be achieved when hCMPCs are grown onto fibronectin-coated polyacrylamide gels with cardiac tissue-like stiffness $(10 \mathrm{kPa})(\mathrm{d}) .{ }^{*}, * * P<0.05$, between $10 \mathrm{kPa}$ and 0.5 and $40 \mathrm{kPa}(n=3)$. Cardiac differentiation is impaired in YAP/TAZ-silenced cells, as demonstrated by cTnT-GFP expression analysis in FACS (e) and immunofluorescence ( $f(n=3)$. A murine cardiac progenitor cell line expressing GFP fluorescent protein under Troponin T cardiac differentiation promoter (cTNT-GFP) can be induced to differentiate when cultured in cardiac differentiation medium, while YAP stable silencing (shYAP) results in a significant reduction in cell differentiation $(g)(n=3)$.

transport inhibitor Leptomycin B (preventing YAP/TAZ shuttling to the cytoplasm; see Supplementary Figure 6b) abolished the ability of hCMPCs to produce vessels in Matrigel vasculogenic environment.

\section{DISCUSSION}

In the adult heart, tissue homeostasis is mainly maintained by resident cardiac stem/progenitor cell proliferation and differentiation throughout the organism lifespan. ${ }^{24,36,37}$ These cells are endowed with the capacity to replenish cardiac tissue by generating new contractile and vascular cells after an ischemic insult, ${ }^{26,38}$ despite their efficiency is still debated. ${ }^{39}$

Tissue remodeling is a complex process occurring after the myocardial infarction, leading to severe modifications in the cardiac ECM nanostructure; ${ }^{19,40}$ the 
a

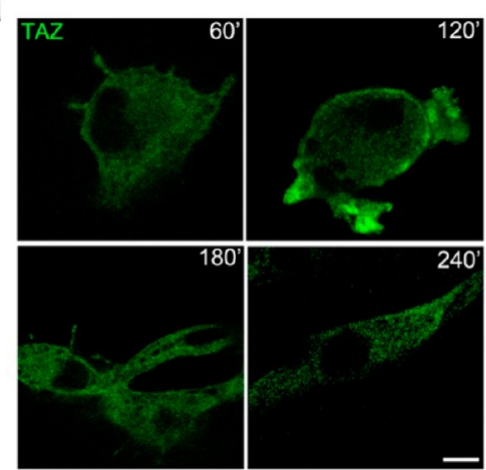

b

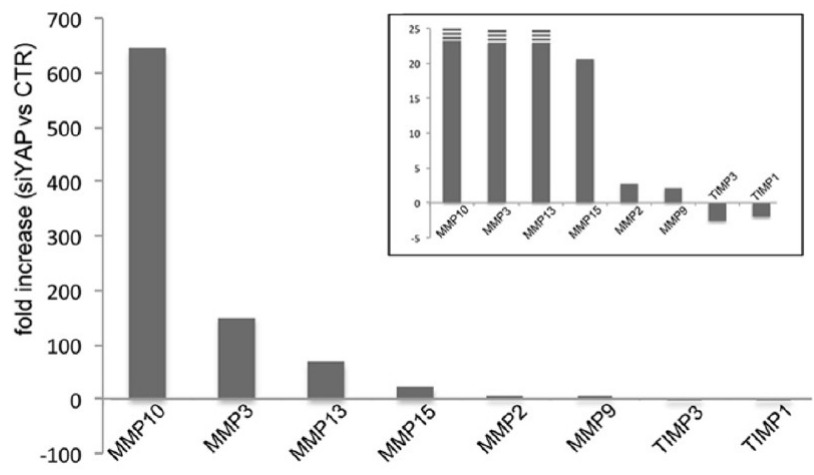

C

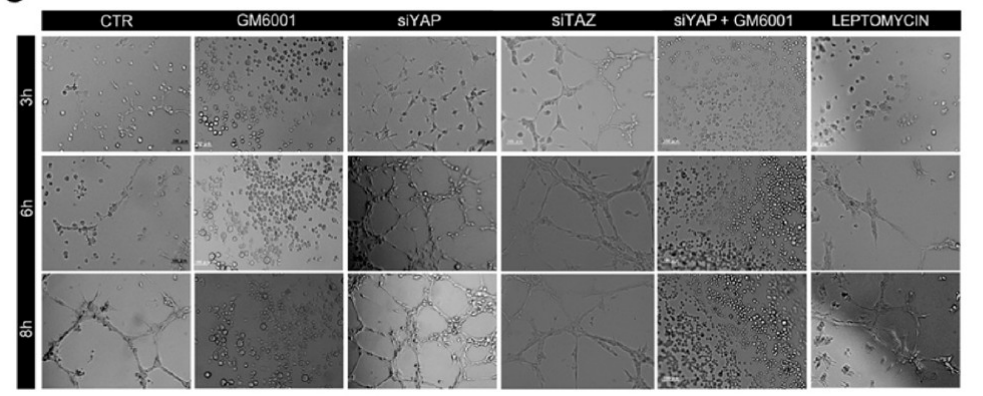

d
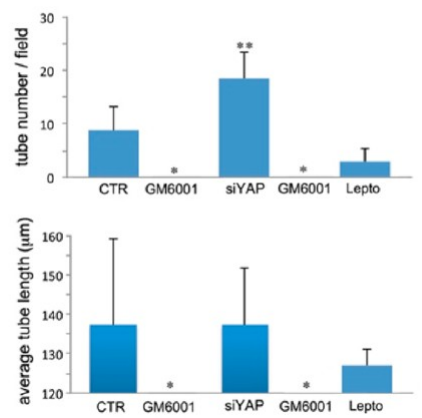

Figure 6. YAP/TAZ control hCMPC endothelial differentiation within soft substrates through metalloproteinase regulation. YAP/TAZ expression was followed in hCMPCs grown onto Matrigel substrate, showing that their expression was exclusively detected in the cytoplasm, as it was expected for such a soft substrate $(E<1 \mathrm{kPa})(\mathrm{a})$. YAP silencing in cardiac progenitor cells (siYAP) (b) resulted in the up-regulation of genes coding for metalloproteinases (MMPs) (c) and the down-regulation of tissue inhibitor of metalloproteinases (TIMPs). Thus, siYAP cells displayed enhanced tubulogenesis in Matrigel (c,d). This event was reverted by treatment with the broad-spectrum MMP inhibitor GM6001. Nuclear transport inhibitor Leptomycin B (Lepto, triggering YAP/TAZ nuclear retention) abolished cell tubulogenesis. These results suggest that YAP/TAZ exert an effect in the cytoplasm of hCMPC and control cell migration in a soft environment by repressing MMP activation. ${ }^{*} P<0.01$ between samples treated or not with GM6001 inhibitor; ${ }^{* * P}<0.05$ between siYAP cells and control.

remodeling is guided by the activity of tissue metalloproteinases and eventually ends with the local compliance increasing from $\approx 10 \mathrm{kPa}$ to $35-70 \mathrm{kPa}$ within 4-6 weeks. $^{14}$ The alteration in matrix stiffness is responsible for the impairment of cardiac contractility following $\mathrm{Ml}$ and eventually hinders stem/progenitor cell differentiation and homing to the infarcted area. ${ }^{28,29}$

The responsiveness of adult and undifferentiated cells to substrate stiffness and topology has been the subject of a number of investigations so far, showing that cell-specific responses can be triggered by the mechanics of the substrate. ${ }^{12,20,30,41,42}$ Moreover, a specific response of tissue resident cardiac progenitor cells to substrate mechanical properties has been highlighted. ${ }^{25,43}$

Recently, cell sensitivity to substrate composition has been associated with the ability of the Hippo pathway downstream effectors YAP and TAZ to act as transcriptional coactivators directly binding to lineage-specific effectors, thus acting as on-off relays in mechano-transduction. ${ }^{18}$ These factors are also responsible for proper cardiac development during fetal life by controlling cardiomyocyte proliferation ${ }^{21}$ and to promote adult cardiomyocyte survival after a myocardial insult. ${ }^{27}$
Here we demonstrate that YAP/TAZ are crucial for cardiac progenitor cell fate decision, as controlled by cardiac- or endothelial-like substrate properties. Moreover, these proteins were also involved in cardiac progenitor cell differentiation when cardiogenic supplements were added to the growth medium. In fact, YAP/TAZ silencing in cardiac progenitor cells results in a significant reduction of the basal level of a number of differentiation transcripts (actn2, adrb1, ckm, kcnq1, mb, myh7, myl2, myl3, myl7, ryr2, tnni3, tnnt2, nat1) and in a clear enhancement in cell proliferation and stem cell antigen-1 (Sca-1) expression. Consistently, the induction of Troponin $\mathrm{T}$ (a protein specifically expressed in mature cardiomyocytes) expression by $10 \mathrm{kPa}$ fibronectin-coated PA gel is impaired by nearly $70 \%$ by YAP/TAZ silencing in these cells. These data expand the knowledge about these proteins being fundamental coactivators of differentiation in a variety of adult stem ${ }^{2-8}$ and pluripotent ${ }^{11}$ cell subsets.

Cardiac cell maturation in vitro and in vivo requires the nuclear activation of specific transcription factors, like GATA-4 and Nkx-2.5. ${ }^{25,38}$ Indeed, we demonstrate the up-regulation of such factors on $10 \mathrm{kPa}$ gels, which 
(a)

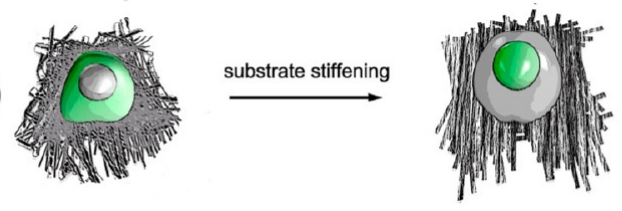

(b)
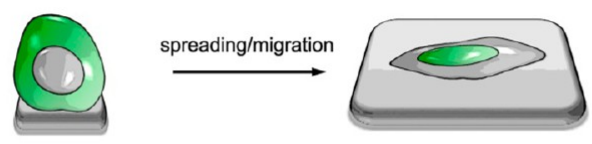

YAPITAZ

(c)
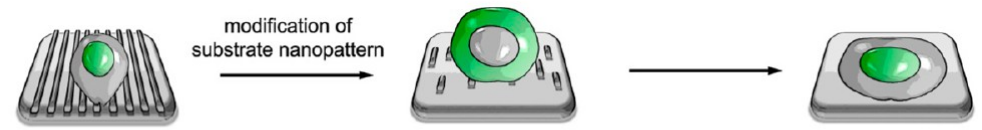

(d)

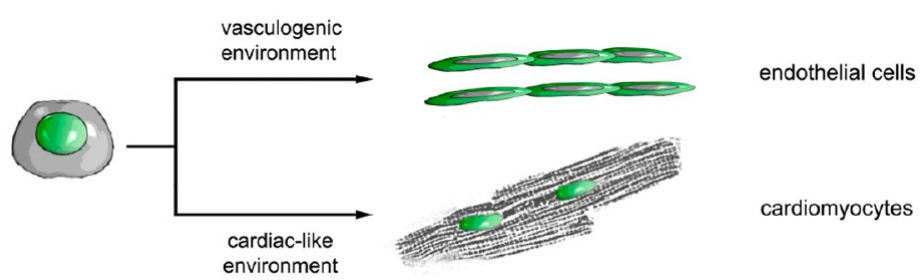

Figure 7. YAP/TAZ control cardiac progenitor cell fate by acting as sensors of extracellular matrix composition. YAP/TAZ activity as transcriptional coactivators is regulated via their phosphorylation in the cytoplasm. Phosphorylated YAP/TAZ are thought to be inactive when retained in the cytoplasm. Nuclear shuttling is triggered in cardiac progenitor cells by substrate stiffening (a), cell spreading or migration (b), and modifications in substrate nanopattern (c). More importantly, the regulation of YAP/TAZ intracellular localization is required for cardiac progenitor cell fate decision (d). When a vasculogenic environment (like Matrigel) is provided, YAP/TAZ are retained in the cytoplasm, while they shuttle to the nucleus when cardiac progenitors are cultured in cardiogenic conditions $(E=10 \mathrm{kPa})$.

are likely to interact with YAP/TAZ coactivators to promote cell differentiation.

Together with our in vivo results showing that YAP and TAZ up-regulation is triggered at the infarction border zone in adult mammalian heart, these data highlight a prompt response of resident cells to ECM stiffening in the postinfarcted myocardium.

On the other side, YAP/TAZ silencing in cardiac progenitors resulted in a massive up-regulation in endothelial-specific genes (edn1, eng, flt1, id1, kdr, pecam1, pgf, tek), thus suggesting a role for these proteins in controlling the switch between cardiac and endothelial lineages in the adult heart.

This evidence was strengthened by a Matrigel-based tube formation assay: in these vasculogenic conditions, where YAP and TAZ showed an exclusive cytoplasmic localization (given to the softness of the material), their silencing enhanced cardiac progenitor migration and tube formation. These data suggest additional roles for YAP/TAZ in the cytoplasm when cells are grown on soft materials $(E<0.7 \mathrm{kPa})$, which will require further investigation. This possibility is corroborated by the use of the selective inhibitor of nuclear transport Leptomycin B, which prevents cell migration and endothelial differentiation in Matrigel.

In turn, we demonstrate that YAP and TAZ act as general mechano-sensors in cardiac progenitor cells: their subcellular localization is modulated by cell spreading and dynamic changes in substrate stiffness and nanostructure. In fact, a gradient in YAP/TAZ nuclear expression can be detected in cardiac progenitors as a result of their ability to spread and organize their cytoskeleton, similarly to what has been previously demonstrated in other cell types. ${ }^{18}$ Interestingly, the evidence that YAP/TAZ expression does not change in cells having the same area but different shapes suggests that cell fate can be controlled by the availability of adhesion sites within the substrate more than by the cell shape itself.

More importantly, our data provide the first evidence for YAP/TAZ shuttling activity between the nucleus and the cytoplasm being promptly activated in response to dynamic modifications in substrate stiffness or nanostructure. By using thermo-responsive materials displaying shape-memory properties, we aimed at mimicking in vitro the modifications in cardiac ECM nanotopography and follow YAP/TAZ response in cardiac progenitors. YAP/TAZ shuttling to the cytoplasm, an event known to precede their inactivation by phosphorylation occurs immediately after a modification in substrate stiffness or nanostructure is imposed by slight temperature changes in thermo-responsive polymers. This result is consistent with previous data showing that perturbations in cell adhesion cause a temporary alteration in YAP/TAZ intracellular localization. ${ }^{17}$ Unfortunately, due to the 
chemical composition of thermo-responsive $\mathrm{PCL}$ polymers displaying shape-memory properties, by this means it is not possible to decouple completely the effect of stiffness from that induced by the modification in nanotopography on cardiac progenitor cells.

YAP/TAZ seem to be required for CPC motility on stiff surfaces, given that their silencing is responsible for a significant reduction in cell migration. While pointing at the different roles exerted by the two Hippo effectors on materials with distinct compliances, these results can also be explained by the different nature of $2 \mathrm{D}$ and $3 \mathrm{D}$ migration, the latter relying on MMP activation. ${ }^{44,45}$ As such, these results would confirm the pivotal function of YAP and TAZ in the switch between mesenchymal and amoeboid migration previously suggested. $^{46}$

\section{CONCLUSIONS}

In conclusion, the data herein, in addition to indicate YAP/TAZ as novel regulators of cardiac differentiation, also outline the multifaceted effects of these factors in determining the cellular response to modifications in substrate nanostructure, altering cell adhesion and spreading, triggering their migration or controlling their differentiation (Figure 7). These results propose YAP/TAZ as a pivotal axis in cardiac progenitor cell interaction with the signals coming from the ECM. They are thus indicated as potential targets to control cardiac progenitor cell fate by materials design.

\section{MATERIALS AND METHODS}

Isolation of Human and Murine Sca-1 ${ }^{+}$Cardiac Progenitor Cells and Preparation of Murine Sca- ${ }^{+}$Cardiac Progenitor Cell Line. Murine Sca- $1^{+}$cardiac progenitor cells were obtained from 6 week male $\mathrm{C} 57 \mathrm{BI} / 6$ mice heart as previously described. ${ }^{43}$ Cells were cultured in DMEM supplemented with $10 \% \mathrm{FCS}, 100 \mathrm{IU} / \mathrm{mL}$ penicillin, $100 \mu \mathrm{g} / \mathrm{mL}$ streptomycin, insulin/transferrin/selenium $1 \mathrm{X}, 300 \mathrm{ng} / \mathrm{mL}$ endothelial growth factor (hereafter referred to as "complete medium"). To obtain a stable murine Sca- $1^{+}$ cardiac progenitor cell line, $10 \mu \mathrm{g}$ of plasmid pCINeo-TERT or control vector $\mathrm{pCINeo}$ was introduced into $\mathrm{Sca}-1^{+}$cardiacderived cells using the calcium phosphate method (Promega, Madison, WI, USA), as previously described. ${ }^{47,48}$

Human Sca- $1^{+}$cardiac progenitor cells (hCMPCs or Sca- $1^{+}$ hCMPCs) were obtained from human biopsies as previously described $^{36}$ and their characterization and differentiation reported. ${ }^{26}$ Briefly, human adult auricles were minced and treated with collagenase. By using magnetic cell sorting (MACS, Miltenyi Biotec), hCMPCs were separated with Sca-1 coupled beads. Cells were cultured in M199 + EGM-2 (3:1), supplemented with $10 \%$ FCS, $0.02 \%$ penicillin/streptomycin, and $0.01 \%$ MEM nonessential amino acids.

For cell confinement studies, cells were cultured onto different fibronectin-coated micropatterned surfaces (Cytoo SA, Grenoble, France). The first set was designed to display square adhesive islands of different surface areas (300, 1024, 2025 , and $10000 \mu \mathrm{m}^{2}$ ). The second set had the same surface area $\left(2000 \mu \mathrm{m}^{2}\right)$ and different shapes (square, round, or triangle). Single cells were allowed to adhere for 3,5 , and $24 \mathrm{~h}$, stained with the appropriate antibodies, and visualized under the confocal microscope.

Cytochalasin D (1 mg/mL) (Sigma, USA), Y27632 (50 $\mu \mathrm{M})$ (WAKO chemicals, Japan), and Blebbistatin (Blebb, $50 \mu \mathrm{M}$ ) pharmacological inhibitors (Merck Biosciences, USA) were dissolved in PBS and added to growth medium (10\% of the final treatment volume) for $4 \mathrm{~h}$. Alternatively, the inhibitors were added for $4 \mathrm{~h}$ to cell growth medium, then the medium was removed and substituted with fresh one. The cells were analyzed after $6 \mathrm{~h}$ recovery.

Preparation and Characterization of Thermo-Responsive Polymers with Shape-Memory Properties. Thermo-responsive polymers were prepared by cross-linking tetra-branched poly- $\varepsilon$-caprolactone $(P C L)$ with acrylate end groups in the presence of linear PCL telechelic diacrylates, according to a previously reported protocol. ${ }^{32}$ Briefly, two-branched and four-branched PCL were synthesized by $\varepsilon$-caprolactone $(\mathrm{CL})$ ring-opening polymerization that was initiated with tetramethylene glycol and pentaerythritol as initiators, respectively. Then, acryloyl chloride was reacted to the end of the branched chains. The structures and the molecular weights were estimated by ${ }^{1} \mathrm{H}$ NMR spectroscopy
(JEOL, Japan) and gel permeation chromatography (JASCO International, Japan). The obtained PCL macromonomers were then dissolved in xylene containing benzoyl peroxide (BPO), and the solution was injected between a glass slide with a $0.2 \mathrm{~mm}$ thick Teflon spacer to prepare the substrate layers. The PCL macromonomers were cured for $180 \mathrm{~min}$ at $80^{\circ} \mathrm{C}$. The mechanical properties of the cross-linked materials were characterized by tensile test (EZ-S 500N, Shimadzu, Japan). The contact angles on the PCL layers were determined by sessile drop method $30 \mathrm{~s}$ after a water drop was placed on the surface at 32 or $37^{\circ} \mathrm{C}$. The contact angles and the mechanical properties of the PCL layers are detailed in Supplementary Figure 1d.

To prepare shape-memory PCL substrates with permanent surface nanopattern, the PCL macromonomer solution was cured between a nanopatterned glass mold and a flat glass slide with a $0.2 \mathrm{~mm}$ thick Teflon spacer for $180 \mathrm{~min}$ at $80^{\circ} \mathrm{C}$. Nanopatterned molding of glass substrates was performed by using an electron beam (EB) lithography system (ELS-7500EX, Elionix, Hachioji, Japan). To program temporary surface patterns, the films were compressed in a thermochamber. A compressive stress of $0.1 \mathrm{MPa}$ was applied to the samples at $37^{\circ} \mathrm{C}$ and maintained for $5 \mathrm{~min}$. The embossing stress was then released at $4{ }^{\circ} \mathrm{C}$ after $10 \mathrm{~min}$ of cooling. Samples had a temporary surface pattern that could be triggered to transition to the permanent surface pattern by heating. Nanopatterned PCL surfaces were observed by AFM (SPM-9500J3, Shimadzu Co., Kyoto, Japan) in noncontact mode using a $\mathrm{Si}_{3} \mathrm{~N}_{4}$ cantilever (spring constant; $42 \mathrm{~N} \mathrm{~m}^{-1}$ ), and the sample temperature was controlled. The nanostructure characterization of the scaffolds is shown in Supplementary Figure 5.

Generation of YAP-GFP and cTnT-GFP Cardiac Progenitor Cells. pEGFPC3-hYAP1 clone was obtained from Addgene nonprofit plasmid repository (ADDGENE, Cambridge, MA, USA). pEGFP-C3-hYAP1bearing colonies were plated on LB agar plates containing $30 \mu \mathrm{g} / \mathrm{mL}$ kanamycin and incubated at $37^{\circ} \mathrm{C}$ overnight. Positive colonies were picked the day after and DNA extracted, purified with QIAGEN plasmid midi purification kit (QIAGEN GmbH, Hilden, Germany) following the manufacturer's instructions, and dissolved in TE buffer $(\mathrm{pH}=7)$. DNA concentration and purity were estimated with Nanodrop ND-1000 (Nanodrop Technologies).

Subsequently, hCMPCs were transiently transfected with pEGFP-C3-hYAP1 $(2 \mu \mathrm{g})$ using SuperFect transfection reagent (QIAGEN GmbH, Hilden, Germany). Briefly, the day before transfection, hCMPCs cells were seeded on 24 multiwell plates and incubated at $37{ }^{\circ} \mathrm{C}$ and $5 \% \mathrm{CO}_{2}$ overnight. On the day of transfection, $2 \mu \mathrm{g}$ of pEGFP-C3-hYAP1 DNA was diluted in cell growth medium containing no serum or antibiotics $(120 \mu \mathrm{L})$. Thereafter, $10 \mu \mathrm{L}$ of SuperFect transfection reagent was added to the DNA solution, mixed, and incubated $10 \mathrm{~min}$ at room 
temperature to allow transfection complex formation. Cell growth medium $(530 \mu \mathrm{L})$ containing serum and antibiotics was added to the reaction tube containing the transfection complexes. hCMPCs were incubated at $37{ }^{\circ} \mathrm{C}$ and $5 \% \mathrm{CO}_{2}$ and after $7 \mathrm{~h}$ medium was replaced with fresh cell growth medium (containing serum and antibiotics). Cells transfected were analyzed $24-72 \mathrm{~h}$ after transfection.

The $\mathrm{pGreenZeo} \mathrm{lentiviral} \mathrm{expression} \mathrm{vector} \mathrm{carrying} \mathrm{the} \mathrm{full-}$ length copGFP gene under the control of mouse- or humanspecific cardiac Troponin (cTnT) promoter was purchased from SBI (System Biosciences Inc., Mountain View, CA; cat nos. SR10013PA-1, SR10012PA-1) and delivered into mammalian cells according to the manufacturer's instructions. The cells were transduced at an approximate multiplicity of infection (MOI) of 20 and incubated at $37^{\circ} \mathrm{C}$ overnight. The following day, half of the culture medium was replaced with fresh medium, and $48 \mathrm{~h}$ after infection, the medium was substituted with fresh one.

Fluorescence-Activated Cell Sorting (FACS) Analysis. FACSCalibur flow cytometer (BD Biosciences, USA) was used to measure the percentage of cTnT-GFP expressing cells and $\mathrm{Sca}-1^{+}$cells. Cells with fluorescence intensity greater than $10^{1}$ were considered to be positive, and the gating was done accordingly. A total of 10000 events were scored. To determine the percentage of Sca- $1^{+}$cells, control and transduced cells were washed in $0.5 \%$ bovine serum albumin in PBS buffer (washing buffer), incubated on ice in the dark with PE/Cy5 anti-mouse Ly-6A/E (Sca-1) (Biolegend Inc., San Diego, CA) for 15 min, and washed twice with cold washing buffer. Data were acquired using Cell Quest software and the analysis performed using the WinMDI 2.9 software.

Transfection of siRNA and shRNA into Cardiac Progenitor Cells. siRNAs targeting YAP or TAZ genes were purchased from Santa Cruz (Santa Cruz Biotechnology Inc., Santa Cruz, California 95060, USA; cat nos. sc-38637 and sc-38568, respectively). Briefly, cells were seeded at $2.5 \times 10^{4}$ cells/well in serum- and antibioticfree Opti-MEM reduced serum medium (Life Technologies) in 24-well plates the day prior to transfection. A mixture of siRNA duplex (1.5 $\mu \mathrm{L} /$ well) and siRNA transfection reagent $(1.5 \mu \mathrm{L} /$ well, sc-29528, Santa Cruz Biotechnology) was formed in siRNA transfection medium (47 $\mu \mathrm{L} /$ well, sc-36868, Santa Cruz Biotechnology) for $15 \mathrm{~min}$ at room temperature. Complexes were added to wells (50 $\mu \mathrm{L} /$ well) containing cells and incubated for $7 \mathrm{~h}$ at $37^{\circ} \mathrm{C}$ in $0.2 \mathrm{~mL}$ of siRNA transfection medium. At the end of the incubation, normal growth medium containing $20 \%$ FBS and $2 X$ antibiotics was added in every well without removing the transfection mixture. Twenty-four hours later, the medium was replaced with fresh normal growth medium. All experiments with silenced cells were performed $48 \mathrm{~h}$ post-transfection in triplicate, and they were repeated three times. A similar procedure was used with scrambled siRNA (control siRNA, sc37007 ) to rule out the occurrence of off-target effects. The data shown are from representative experiments.

Stable yap gene silencing was achieved in murine CPC cell line ( $\mathrm{mCPCs}$ ) by transducing them with YAP shRNA lentiviral particles $(\mathrm{MOI}=20$, Santa Cruz Biotechnology, sc-38637-V). Twenty-four hours after the infection, fresh medium was added and infected cells selected with $10 \mu \mathrm{g} / \mathrm{mL}$ puromycin (Santa Cruz) for 1 month. Puromycin-resistant cells were transduced with the lentiviral construct encoding for copGFP under the control of mouse cTnT promoter as above-described and induced to differentiate toward cardiac lineage according to a previously reported protocol. ${ }^{36}$ Two weeks later, stably selected transduced cells were analyzed for eGFP expression by flow cytometry.

Matrigel Tubulogenesis Assay. BD Matrigel basement membrane matrix growth factor reduced, phenol red free (BD Biosciences, USA), was prepared for the thick gel method according to the manufacturer's instructions. Briefly, $40 \mu \mathrm{L}$ of Matrigel solution containing or not GM6001 inhibitor (100 nM, EMD Millipore Corporation, Billerica, MA, USA) were added on 96 cooled wells (IWAKI, Japan). Thereafter, the plates were placed at $37^{\circ} \mathrm{C}$ for $30 \mathrm{~min}$ in order to enable proper gelation. Afterward, $3.0 \times 10^{4} \mathrm{hCMPCs}$ were suspended in $100 \mu \mathrm{L}$ complete medium containing or not GM6001 inhibitor (100 nM) and plated on top of the gel. At each relevant time point $(3,6$, and $8 \mathrm{~h})$, brightfield pictures were taken using an Olympus IX71 microscope equipped with a digital camera. The number and length of tubelike structures were measured by manual tracing of vessels by ImageJ software (version 1.46; National Institutes of Health, Bethesda, MD). Average values were determined by counting 10 fields per sample at different time points from three independent experiments. At the same time points, cells were fixed and analyzed by immunofluorescence by staining them with anti-YAP antibody as described below.

Preparation of Polyacrylamide Films with Physiological Stiffness. Polyacrylamide gels with stiffness values of $0.5,10$, and $40 \mathrm{kPa}$ were prepared as described, ${ }^{20}$ with minor modifications. Glass round coverslips (Matsunami glass, Japan) were amino-silanized using aminopropyltriethoxysilane (APTES), whereas square glass slides were fluoro-silanized by the use of fluorosilane (Trichloro ( $1 \mathrm{H}, 2 \mathrm{H}, 2 \mathrm{H}$-perfluorooctyl)silane) (Sigma Aldrich). Afterward, PA gels were prepared by the addition of the adequate proportion of $40 \%(\mathrm{w} / \mathrm{v})$ acrylamide stock solution (Sigma, USA) to $2 \%(\mathrm{w} / \mathrm{v})$ bisacrylamide ( $N, N^{\prime}$-methylenebisacrylamide) (Tokyo Chemical Industry, Japan) stock solution and PBS, toward achieving the desired stiffness (Young's modulus, $E$ ), according to the reported protocol. Polymerizing catalysts were added (10\% (wv) ammonium persulfate (APS) at $1 \%$ of final volume and tetramethylethylenediamine (TEMED) at $0.1 \%$ final volume). The mixture was poured between the fluoro-silanized glass slide and aminosilanized coverslip for $30 \mathrm{~min}$, after which the PA gel was attached to the amino-silanized coverslip.

Finally, PA gels were functionalized with collagen $(100 \mu \mathrm{g} / \mathrm{mL})$ by the attachment to $0.2 \mathrm{mg} / \mathrm{mL}$ sulfosuccinimidyl-6-(4'-azido$2^{\prime}$-nitrophenylamino)hexanoate (sulfo-SANPAH; Pierce Biotechnology) covalently bound to the gel surface by photoactivation.

Preparation of Photoactivated Patterned Surfaces for Cell Migration. Photocleavable 1-[5-methoxy-2-nitro-4-(trimethoxysilylpropylphenyl)]ethyl $N$-succinimidyl carbonate was covalently bound to an activated glass surface and further coupled to a PEG monoamine (PEG12k-NH). ${ }^{49}$ After thoroughly washing the substrates, a passivated glass surface was obtained, on which cells were not able to adhere. ${ }^{34}$ By introducing a photomask in the field diaphragm of an inverted microscope, equipped with an HBO mercury arc lamp (Olympus) and focusing UV light through an objective (UPlanSApo $10 \times / 0.4$, Olympus) with a wavelength of $365 \mathrm{~nm}$ on the photoactive substrate and exposing it with a dose of $10 \mathrm{~J} / \mathrm{cm}^{2}$, the PEG12k-NH is cleaved off. Thereby, the surface at the irradiated regions became cell-adhesive. Subsequently, YAP-GFP hCMPC cells at a density of $3.5 \times 10^{4}$ cells $/ \mathrm{cm}^{2}$ were seeded on the patterned substrates. One hour after seeding, the medium was replenished to remove nonattached cells. After an incubation time of $3 \mathrm{~h}$, a second UV exposure of the whole substrate with a dose of $10 \mathrm{~J} / \mathrm{cm}^{2}$ released the confinement, initiating cell migration. In order to rule out the possibility that the UV irradiation itself could be the major driving force of the nuclear translocation of YAP, single cardiac progenitor cells were first seeded onto the micropatterned array of $900 \mu \mathrm{m}^{2}$ circular adhesive islands and, after $3 \mathrm{~h}$ incubation, exposed to the UV irradiation. However, in this case, the substrate was irradiated through an array-pattern photomask in order to selectively expose UV to the regions where cells were attached but not to the surrounding regions. The procedure for aligning the primary and secondary irradiation regions was previously reported by our group. ${ }^{50}$ Thus cells exposed to UV light were not able to start migrating since the surrounding regions remained non-cell-adhesive. The cells were fixed with $4 \%$ paraformaldehyde at $2 \mathrm{~h}$ after the secondary irradiation and the location of YAP was examined by staining the cells with antiYAP antibody and DAPI.

Time-Lapse Microscopy and Migration Assay. Single cell migration at multiple positions $(n=10)$ was monitored with phase contrast and fluorescent time-lapse imaging on an automated inverted microscope (XI81; Olympus) equipped with a chargecoupled device camera (Retiga-EXi, Q-Imaging), motorized stage (Molecular Devices), and a heating chamber (INUeONleF1, Tokai Hit) controlled by MetaMorph (Molecular Devices) software. The time frame between images was set at $5 \mathrm{~min}$, and cell 
migration induced between frames 4 and 5 . The acquisition was protracted up to $150 \mathrm{~min}$ in total. Cell migration was quantified by ImageJ software. Briefly, single cells were manually outlined, the center of mass calculated, and the point-to-point displacement in the center of mass obtained by Microsoft Excel analysis.

Immunofluorescence Staining and Confocal Microscopy Analysis. Cells seeded on TCPS dishes, PCL layers, photopatterned surfaces, or PA gels were fixed with 4\% paraformaldehyde in PBS for 30 min at $4{ }^{\circ} \mathrm{C}$ and permeabilized with $0.1 \%$ Triton X-100 (SigmaAldrich) in PBS for $2 \mathrm{~min}$ at room temperature. Cells were incubated with antibodies against YAP, TAZ, GATA-4, Tbx-5, cardiac Troponin T-C (cTnT) (1:200; Santa Cruz Biotechnology), ki67 (ABCAM), vinculin, alpha sarcomeric actinin (sarc actinin) (Sigma-Aldrich), and TurboGFP (Evrogen JSC, Moscow, Russia) for $2 \mathrm{~h}$ at room temperature. The appropriate fluorophoreconjugated secondary antibodies were as follows: Alexa Fluor 488 goat-anti-rabbit, 546 goat-anti-mouse, and 405 donkeyanti-goat (Invitrogen Corp.). Nuclei were counterstained with 4-6-diamidino-2-phenylindole (DAPI; Sigma Aldrich). Secondary antibodies in the absence of a specific primary antibody were used to exclude the occurrence of unspecific signals. The images were taken using a Leica SF5 laser scanner confocal microscope, after excitation at 405, 488, and $543 \mathrm{~nm}$ wavelengths for blue, green, and red channel acquisition, respectively. All pictures are representative of at least three independent experiments.

Protein Extraction and Western Blot. Cell pellets were lysed with ice-cold RIPA buffer (Sigma-Aldrich) containing protease inhibitor cocktail (Thermo Scientific, Asheville, NC) and protein concentration determined using Quick Start Bradford Protein Assay (Bio-Rad, Hercules, CA, USA) and a NanoDrop 1000 spectrophotometer (Thermo Scientific). Forty micrograms of protein lysate was loaded on a 10\% SDS-PAGE precast gel (BioRad) and then transferred onto polyvinylidene difluoride (PVDF) filters (Bio-Rad). Filters were blocked with $1 \%$ casein solution for $45 \mathrm{~min}$ and probed with anti-YAP, anti-TAZ antibodies, or anti- $\beta$ tubulin (1:300, Santa Cruz) diluted in Tris-buffered saline-1\% casein for $2 \mathrm{~h}$ at room temperature.

Membranes were probed with 1:3000 diluted horseradish peroxidase (HRP)-conjugated secondary antibodies (Sigma) for $1 \mathrm{~h}$ and visualized by enhanced chemoluminescence assay (Pierce). Proteins were visualized using Fuji Medical X-ray film, and density of bands was determined using ImageJ software and corrected for loading.

AlamarBlue Assay. The cells were seeded at low density $\left(2 \times 10^{3}\right.$ cells per $\left.\mathrm{cm}^{2}\right)$ in complete medium with $10 \%$ AlamarBlue onto PA gels. Cell adhesion on the substrates was measured by AlamarBlue at $8 \mathrm{~h}$ time point to rule out the occurrence of cell proliferation. Fluorescence intensity was measured by ARVO MX1420 multilabel counter (Perkin-Elmer, Waltham, MA, USA) at F544/F590. Intensity readings were normalized by background intensity and calculated as percentage of the intensity obtained for TCPS at $8 \mathrm{~h}$.

RNA Extraction and Real-Time PCR Array. Total RNA was extracted by TRIZOL Reagent (Gibco BRL). Retrotranscription was carried out with $2 \mathrm{mg}$ of RNA for each sample using reverse transcription (RT) Moloney murine leukemia virus (Invitrogen Corp.) in the presence of random hexamers. Real-time PCR was used to determine the expression profile of key genes involved in different signal transduction pathways in human cardiac progenitors. After synthesis of first-strand cDNAs, RT-PCR $(n=3)$ was performed with a 7500 real-time PCR system (Applied Biosystems) using the RT2 Profiler PCR arrays and human extracellular matrix and adhesion molecules (PAHS-013A, Qiagen), cardiac (iPHS-102), and endothelial differentiation (PAHS-024Z) with the $\mathrm{RT}^{2}$ SYBR green/ROX PCR master mix (Qiagen) according to the manufacturer's protocol. The threshold cycle $\left(C_{t}\right)$ is defined as the fractional cycle number at which the fluorescence reaches 10-fold standard deviation of the baseline (from cycle 3 to cycle 12). The specificity of the SYBR PCR signal was confirmed by melt curve analysis.

Scanning Electron Microscopy (SEM) Analysis. hCMPCs were grown for $24 \mathrm{~h}$ on nanopatterned surfaces at $32{ }^{\circ} \mathrm{C}$. Therefore, the samples were switched to $37^{\circ} \mathrm{C}$ for 90 and $180 \mathrm{~min}$. The samples were fixed in 4\% PFA for $15 \mathrm{~min}$ at room temperature, dried under flow laminar hood, prepared for scanning electron microscopy analysis as previously reported, ${ }^{30}$ and examined using Hitachi S-4800 low-voltage scanning electron microscope.

Experimental Model of Myocardial Infarction. All the in vivo experiments were carried out in accordance with the Guide for the Care and Use of Laboratory Animals, following the approval by the Animal Ethical Experimentation Committee, Leiden University. Myocardial infarction was induced by ligation of the left coronary artery in C57BL/6 mice (Charles River Laboratories) under isoflurane anesthesia $(n=6)$. The mice were sacrificed $3,5,7,14$, and 36 days after MI, and immunohistochemistry analysis was performed as described elsewhere. ${ }^{26}$

Statistical Analysis. Statistical analyses were performed using Microsoft Excel software. The results are shown as mean \pm standard deviation (SD). Student's $t$ test was used to generate all $P$ values. $P<0.05$ was considered statistically significant. The number of replicated experiments performed is given as $n$.

Conflict of Interest: The authors declare no competing financial interest.

Acknowledgment. The present work was supported by the Japan Society for the Promotion of Science (JSPS) through the "Funding Program for World-Leading Innovative R\&D on Science and Technology (FIRST Program)", by the World Premiere International (WPI) Research Center Initiative, by the "Nanotechnology Network Project" of the Ministry of Education, Culture, Sports, Science and Technology (MEXT), Japan and by the European Regional Development Fund - Project FNUSA-ICRC (No. CZ.1.05/1.1.00/02.0123). The contribution of The Netherlands Institute for Regenerative Medicine (NIRM) and FINSKIN Project no. 273689 from the Academy of Finland is also gratefully acknowledged. The authors are grateful to Dr. Isabel Amaral for critical discussion, Mr. Sjoerd Duim for immunohistochemistry analysis, and Mr. Gianluca Discenza and Mr. Massimiliano Massarelli for the advice with statistical analysis. Moreover, the authors would like to thank Dr. Elena Romano and the Center for Advanced Microscopy "Patrizia Albertano" of the University of Rome "Tor Vergata" for confocal image analysys. P.P.Ó. was supported by Ciência 2007 and the FCTG Grant PTDC/SAU-ORG/118297/2010. C.E.L. was supported by the Academy of Finland. Financial supporters had no role in study design, data collection and analysis, decision to publish, or preparation of the manuscript.

Supporting Information Available: Supplementary Figures 1-6 provide graphical data supporting the observed results, as mentioned throughout the text in the Results section. Supplementary movies 1 and 2 consist of animations of material preparation and characterization. This material is available free of charge via the Internet at http://pubs.acs.org.

\section{REFERENCES AND NOTES}

1. Hong, W.; Guan, K. L. The YAP and TAZ Transcription Coactivators: Key Downstream Effectors of the Mammalian Hippo Pathway. Semin. Cell Dev. Biol. 2012, 23, 785-793.

2. Zhao, B.; Lei, Q. Y.; Guan, K. L. The Hippo-YAP Pathway: New Connections between Regulation of Organ Size and Cancer. Curr. Opin. Cell Biol. 2008, 20, 638-646.

3. Hong, J. H.; Yaffe, M. B. TAZ: A Beta-Catenin-like Molecule That Regulates Mesenchymal Stem Cell Differentiation. Cell Cycle 2006, 5, 176-179.

4. Hong, J. H.TAZ, a Transcriptional Modulator of Mesenchymal Stem Cell Differentiation. Science 2005, 309, 1074-1078.

5. Varelas, X.; Sakuma, R.; Samavarchi-Tehrani, P.; Peerani, R.; Rao, B. M.; Dembowy, J.; Yaffe, M. B.; Zandstra, P. W.; Wrana, J. L. TAZ Controls Smad Nucleocytoplasmic Shuttling and Regulates Human Embryonic Stem-Cell Self-Renewal. Nat. Cell Biol. 2008, 10, 837-848.

6. Camargo, F. D.; Gokhale, S.; Johnnidis, J. B.; Fu, D.; Bell, G. W.; Jaenisch, R.; Brummelkamp, T. R. YAP1 Increases Organ Size and Expands Undifferentiated Progenitor Cells. Curr. Biol. 2007, 17, 2054-2060.

7. Cao, X.; Pfaff, S. L.; Gage, F. H. YAP Regulates Neural Progenitor Cell Number via the TEA Domain Transcription Factor. Genes Dev. 2008, 22, 3320-3334.

8. Zhang, H.; Pasolli, H. A.; Fuchs, E. Yes-Associated Protein (YAP) Transcriptional Coactivator Functions in Balancing 
Growth and Differentiation in Skin. Proc. Natl. Acad. Sci. U.S.A. 2011, 108, 2270-2275.

9. Watt, K. I.; Judson, R.; Medlow, P.; Reid, K.; Kurth, T. B.; Burniston, J. G.; Ratkevicius, A.; Bari, C. D.; Wackerhage, H. Yap Is a Novel Regulator of C2C12 Myogenesis. Biochem. Biophys. Res. Commun. 2010, 393, 619-624.

10. Jeong, H.; Bae, S.; An, S. Y.; Byun, M. R.; Hwang, J. H.; Yaffe, M. B.; Hong, J. H.; Hwang, E. S. TAZ as a Novel Enhancer of MyoD-Mediated Myogenic Differentiation. FASEB J. 2010, 24, 3310-3320.

11. Qin, H.; Blaschke, K.; Wei, G.; Ohi, Y.; Blouin, L.; Qi, Z.; Yu, J.; Yeh, R.-F.; Hebrok, M.; Ramalho-Santos, M. Transcriptional Analysis of Pluripotency Reveals the Hippo Pathway as a Barrier to Reprogramming. Hum. Mol. Genet. 2012, 21, 2054-2067.

12. Engler, A. J.; Sen, S.; Sweeney, H. L.; Discher, D. E. Matrix Elasticity Directs Stem Cell Lineage Specification. Cell 2006, 126, 677-689.

13. Romanazzo, S.; Forte, G.; Ebara, M.; Uto, K.; Pagliari, S.; Aoyagi, T.; Traversa, E.; Taniguchi, A. Substrate Stiffness Affects Skeletal Myoblast Differentiation in Vitro. Sci. Technol. Adv. Mater. 2012, 13, 064211.

14. Engler, A. J.; Carag-Krieger, C.; Johnson, C. P.; Raab, M.; Tang, H. Y.; Speicher, D. W.; Sanger, J. W.; Sanger, J. M.; Discher, D. E. Embryonic Cardiomyocytes Beat Best on a Matrix with Heart-like Elasticity: Scar-like Rigidity Inhibits Beating. J. Cell Sci. 2008, 121, 3794-3802.

15. Musah, S.; Morin, S. A.; Wrighton, P. J.; Zwick, D. B.; Jin, S.; Kiessling, L. L. Glycosaminoglycan-Binding Hydrogels Enable Mechanical Control of Human Pluripotent Stem Cell Self-Renewal. ACS Nano 2012, 6, 10168-10177.

16. Zhao, B.; Wei, X.; Li, W.; Udan, R. S.; Yang, Q.; Kim, J.; Xie, J.; Ikenoue, T.; Yu, J; Li, L.; et al. Inactivation of YAP Oncoprotein by the Hippo Pathway Is Involved in Cell Contact Inhibition and Tissue Growth Control. Genes Dev. 2007, 21, 2747-2761.

17. Zhao, B.; Li, L.; Wang, L.; Wang, C. Y.; Yu, J.; Guan, K. L. Cell Detachment Activates the Hippo Pathway via Cytoskeleton Reorganization To Induce Anoikis. Genes Dev. 2012, 26, 54-68.

18. Dupont, S.; Morsut, L.; Aragona, M.; Enzo, E.; Giulitti, S.; Cordenonsi, M.; Zanconato, F.; Le Digabel, J.; Forcato, M.; Bicciato, S.; et al. Role of YAP/TAZ in Mechanotransduction. Nature 2011, 474, 179-183.

19. Spinale, F. G. Myocardial Matrix Remodeling and the Matrix Metalloproteinases: Influence on Cardiac Form and Function. Physiol. Rev. 2007, 87, 1285-1342.

20. Engler, A. J.; Rehfeldt, F.; Sen, S.; Discher, D. E. Microtissue Elasticity: Measurements by Atomic Force Microscopy and Its Influence on Cell Differentiation. In Methods in Cell Biology; Yu-Li, W., Dennis, E. D., Eds.; Academic Press: New York, 2007; Vol. 83, pp 521-545.

21. von Gise, A.; Lin, Z.; Schlegelmilch, K.; Honor, L. B.; Pan, G. M.; Buck, J. N.; Ma, Q.; Ishiwata, T.; Zhou, B.; Camargo, F. D.; et al. YAP1, the Nuclear Target of Hippo Signaling, Stimulates Heart Growth through Cardiomyocyte Proliferation but Not Hypertrophy. Proc. Natl. Acad. Sci. U.S.A. 2012, 109, 2394-2399.

22. Xin, M.; Kim, Y.; Sutherland, L. B.; Qi, X.; McAnally, J.; Schwartz, R. J.; Richardson, J. A.; Bassel-Duby, R.; Olson, E. N. Regulation of Insulin-like Growth Factor Signaling by Yap Governs Cardiomyocyte Proliferation and Embryonic Heart Size. Sci. Signal. 2011, 4, ra70.

23. Heallen, T.; Zhang, M.; Wang, J.; Bonilla-Claudio, M.; Klysik, E.; Johnson, R. L.; Martin, J. F. Hippo Pathway Inhibits Wnt Signaling To Restrain Cardiomyocyte Proliferation and Heart Size. Science 2011, 332, 458-461.

24. Bergmann, O.; Bhardwaj, R. D.; Bernard, S.; Zdunek, S.; Barnabe-Heider, F.; Walsh, S.; Zupicich, J.; Alkass, K.; Buchholz, B. A.; Druid, H.; et al. Evidence for Cardiomyocyte Renewal in Humans. Science 2009, 324, 98-102.

25. Pagliari, S.; Vilela-Silva, A. C.; Forte, G.; Pagliari, F.; Mandoli, C.; Vozzi, G.; Pietronave, S.; Prat, M.; Licoccia, S.; Ahluwalia, A.; et al. Cooperation of Biological and Mechanical Signals in Cardiac Progenitor Cell Differentiation. Adv. Mater. 2011, 23, 514-518.
26. Forte, G.; Pietronave, S.; Nardone, G.; Zamperone, A.; Magnani, E.; Pagliari, S.; Pagliari, F.; Giacinti, C.; Nicoletti, C.; Musaró, A.; et al. Human Cardiac Progenitor Cell Grafts as Unrestricted Source of Supernumerary Cardiac Cells in Healthy Murine Hearts. Stem Cells 2011, 29, 2051-2061.

27. Del Re, D. P.; Yang, Y.; Nakano, N.; Cho, J.; Zhai, P.; Yamamoto, T.; Zhang, N.; Yabuta, N.; Nojima, H.; Pan, D.; et al. Yes-Associated Protein Isoform 1 (Yap1) Promotes Cardiomyocyte Survival and Growth To Protect Against Myocardial Ischemic Injury. J. Biol. Chem. 2013, 288, 39773988.

28. Breitbach, M.; Bostani, T.; Roell, W.; Xia, Y.; Dewald, O.; Nygren, J. M.; Fries, J. W. U.; Tiemann, K.; Bohlen, H.; Hescheler, J.; et al. Potential Risks of Bone Marrow Cell Transplantation into Infarcted Hearts. Blood 2007, 110, 1362-1369.

29. Murry, C. E.; Soonpaa, M. H.; Reinecke, H.; Nakajima, H.; Nakajima, H. O.; Rubart, M.; Pasumarthi, K. B.; Virag, J. I.; Bartelmez, S. H.; Poppa, V.; et al. Haematopoietic Stem Cells Do Not Transdifferentiate into Cardiac Myocytes in Myocardial Infarcts. Nature 2004, 428, 664-668.

30. Forte, G.; Pagliari, S.; Ebara, M.; Uto, K.; Kal Van Tam, J.; Romanazzo, S.; Escobedo-Lucea, C.; Romano, E.; Di Nardo, P.; Traversa, E.; et al. Substrate Stiffness Modulates Gene Expression and Phenotype in Neonatal Cardiomyocytes in Vitro. Tissue Eng., Part A 2012, 18, 1837-1848.

31. Engler, A. J.; Griffin, M. A.; Sen, S.; Bönnemann, C. G.; Sweeney, H. L.; Discher, D. E. Myotubes Differentiate Optimally on Substrates with Tissue-like Stiffness: Pathological Implications for Soft or Stiff Microenvironments. J. Cell Biol. 2004, 166, 877-887.

32. Ebara, M.; Uto, K.; Idota, N.; Hoffman, J. M.; Aoyagi, T. Shape-Memory Surface with Dynamically Tunable NanoGeometry Activated by Body Heat. Adv. Mater. 2012, 24, 273-278.

33. McBeath, R.; Pirone, D. M.; Nelson, C. M.; Bhadriraju, K.; Chen, C. S. Cell Shape, Cytoskeletal Tension, and RhoA Regulate Stem Cell Lineage Commitment. Dev. Cell 2004, 6, 483-495.

34. Rolli, C. G.; Nakayama, H.; Yamaguchi, K.; Spatz, J. P.; Kemkemer, R.; Nakanishi, J. Switchable Adhesive Substrates: Revealing Geometry Dependence in Collective Cell Behavior. Biomaterials 2012, 33, 2409-2418.

35. Tateishi, K.; Ashihara, E.; Takehara, N.; Nomura, T.; Honsho, S.; Nakagami, T.; Morikawa, S.; Takahashi, T.; Ueyama, T.; Matsubara, H.; et al. Clonally Amplified Cardiac Stem Cells Are Regulated by Sca-1 Signaling for Efficient Cardiovascular Regeneration. J. Cell Sci. 2007, 120, 1791-1800.

36. Smits, A. M.; van Vliet, P.; Metz, C. H.; Korfage, T.; Sluijter, J. P. G.; Doevendans, P. A.; Goumans, M.-J. Human Cardiomyocyte Progenitor Cells Differentiate into Functional Mature Cardiomyocytes: An In Vitro Model for Studying Human Cardiac Physiology and Pathophysiology. Nat. Protoc. 2009, 4, 232-243.

37. Kajstura, J.; Hosoda, T.; Bearzi, C.; Rota, M.; Maestroni, S.; Urbanek, K.; Leri, A.; Anversa, P. The Human Heart: A SelfRenewing Organ. Clin. Transl. Sci. 2008, 1, 80-86.

38. Bearzi, C.; Rota, M.; Hosoda, T.; Tillmanns, J.; Nascimbene, A.; De Angelis, A.; Yasuzawa-Amano, S.; Trofimova, I.; Siggins, R. W.; LeCapitaine, N.; et al. Human Cardiac Stem Cells. Proc. Natl. Acad. Sci. U.S.A. 2007, 104, 14068-14073.

39. Forte, G.; Pagliari, S.; Pagliari, F.; Ebara, M.; Nardo, P.; Aoyagi, T. Towards the Generation of Patient-Specific Patches for Cardiac Repair. Stem Cell Rev. Rep. 2013, 9, 313-325.

40. Liehn, E. A.; Postea, O.; Curaj, A.; Marx, N. Repair after Myocardial Infarction, Between Fantasy and Reality: The Role of Chemokines. J. Am. Coll. Cardiol. 2011, 58, 23572362.

41. Jacot, J. G.; McCulloch, A. D.; Omens, J. H. Substrate Stiffness Affects the Functional Maturation of Neonatal Rat Ventricular Myocytes. Biophys. J. 2008, 95, 3479-3487.

42. Bhana, B.; Iyer, R. K.; Chen, W. L. K.; Zhao, R.; Sider, K. L.; Likhitpanichkul, M.; Simmons, C. A.; Radisic, M. Influence of Substrate Stiffness on the Phenotype of Heart Cells. Biotechnol. Bioeng. 2010, 105, 1148-1160. 
43. Forte, G.; Carotenuto, F.; Pagliari, F.; Pagliari, S.; Cossa, P.; Fiaccavento, R.; Ahluwalia, A.; Vozzi, G.; Vinci, B.; Serafino, A.; et al. Criticality of the Biological and Physical Stimuli Array Inducing Resident Cardiac Stem Cell Determination. Stem Cells 2008, 26, 2093-2103.

44. Sabeh, F.; Shimizu-Hirota, R.; Weiss, S. J. Protease-Dependent versus -Independent Cancer Cell Invasion Programs: Three-Dimensional Amoeboid Movement Revisited. J. Cell Biol. 2009, 185, 11-19.

45. Doyle, A. D.; Wang, F. W.; Matsumoto, K.; Yamada, K. M. One-Dimensional Topography Underlies ThreeDimensional Fibrillar Cell Migration. J. Cell Biol. 2009, 184, 481-490.

46. Even-Ram, S.; Yamada, K. M. Cell Migration in 3D Matrix. Curr. Opin. Cell Biol. 2005, 17, 524-532.

47. Forte, G.; Franzese, O.; Pagliari, S.; Pagliari, F.; Di Francesco, A. M.; Cossa, P.; Laudisi, A.; Fiaccavento, R.; Minieri, M.; Bonmassar, E.; et al. Interfacing Sca-1(pos) Mesenchymal Stem Cells with Biocompatible Scaffolds with Different Chemical Composition and Geometry. J. Biomed. Biotechnol. 2009, 910610.

48. Freire, A. G.; Nascimento, D. S.; Forte, G.; Valente, M.; Resende, T. P.; Pagliari, S.; Abreu, C.; Carvalho, I.; Di Nardo, P.; Pinto-do-Ó, P. Stable phenotype and function of immortalized Lin-Sca-1+ cardiac progenitor cells in longterm culture: a step closer to standardization. Stem Cells Dev. 2013, DOI: 10.1089/scd.2013.0305.

49. Kikuchi, Y.; Nakanishi, J.; Nakayama, H.; Shimizu, T.; Yoshino, Y.; Yamaguchi, K.; Yoshida, Y.; Horiike, Y. Grafting Poly(ethylene glycol) to a Glass Surface via a Photocleavable Linker for Light-Induced Cell Micropatterning and Cell Proliferation Control. Chem. Lett. 2008, 37, 1062-1063.

50. Nakanishi, J.; Kikuchi, Y.; Inoue, S.; Yamaguchi, K.; Takarada, T.; Maeda, M. Spatiotemporal Control of Migration of Single Cells on a Photoactivatable Cell Microarray. J. Am. Chem. Soc. 2007, 129, 6694-6695. 